\title{
A Recursive Formula for the Convolution Sum of Divisor Functions
}

\author{
by \\ mantu \\ Greg Doyle, B.Math (Carleton University) \\ A thesis submitted to \\ the Faculty of Graduate and Postdoctoral Affairs \\ in partial fulfillment of \\ the requirements for the degree of \\ Master of Science
}

School of Mathematics and Statistics

Ottawa-Carleton Institute for Mathematics and Statistics

Carleton University

Ottawa, Ontario, Canada

(C)Copyright

2011, Greg Doyle 
Library and Archives

Canada

Published Heritage

Branch

395 Wellington Street

Ottawa ON K1A ON4

Canada
Bibliothèque et

Archives Canada

Direction du

Patrimoine de l'édition

395, rue Wellington

Ottawa ON K1A ON4

Canada
Your file Votre reférence

ISBN: 978-0-494-79612-2

Our file Notre référence

ISBN: $978-0-494-79612-2$
NOTICE:

The author has granted a nonexclusive license allowing Library and Archives Canada to reproduce, publish, archive, preserve, conserve, communicate to the public by telecommunication or on the Internet, loan, distribute and sell theses worldwide, for commercial or noncommercial purposes, in microform, paper, electronic and/or any other formats.

The author retains copyright ownership and moral rights in this thesis. Neither the thesis nor substantial extracts from it may be printed or otherwise reproduced without the author's permission.

\begin{abstract}
AVIS:
L'auteur a accordé une licence non exclusive permettant à la Bibliothèque et Archives Canada de reproduire, publier, archiver, sauvegarder, conserver, transmettre au public par télécommunication ou par l'Internet, prêter, distribuer et vendre des thèses partout dans le monde, à des fins commerciales ou autres, sur support microforme, papier, électronique et/ou autres formats.
\end{abstract}

L'auteur conserve la propriété du droit d'auteur et des droits moraux qui protège cette thèse. $\mathrm{Ni}$ la thèse ni des extraits substantiels de celle-ci ne doivent être imprimés ou autrement reproduits sans son autorisation.
In compliance with the Canadian Privacy Act some supporting forms may have been removed from this thesis.

While these forms may be included in the document page count, their removal does not represent any loss of content from the thesis.
Conformément à la loi canadienne sur la protection de la vie privée, quelques formulaires secondaires ont été enlevés de cette thèse.

Bien que ces formulaires aient inclus dans la pagination, il n'y aura aucun contenu manquant.

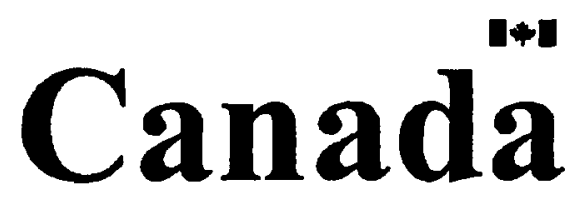




\section{Abstract}

A series of the form $\sum_{n=1}^{\infty} f(n) \frac{q^{n}}{1-q^{n}}$, with $|q|<1$ for complex $q$ is known as a Lambert series. We consider the product of two Lambert series $\left(\sum_{n=1}^{\infty} n^{a} \frac{q^{n}}{1-q^{n}}\right)\left(\sum_{m=1}^{\infty} m^{b} \frac{q^{m}}{1-q^{m}}\right)$, for odd positive integers $a$ and $b$. This product can be written as a formal power series with coefficients $\sum_{m=1}^{n-1} \sigma_{a}(m) \sigma_{b}(n-m)$, a convolution sum involving divisor functıons. A recent theorem by Alaca, Alaca, McAtee and Williams [2, p 7] gives a different expression for the product of two Lambert series. We rewrite their expression as a formal power series and equate those coefficients with $\sum_{m=1}^{n-1} \sigma_{a}(m) \sigma_{b}(n-m)$. By doing so, we are able to derive a recursive formula for this convolution sum. 


\section{Acknowledgements}

First, I must give very special thanks to Dr. Saban Alaca. He was kind enough to agree to be my thesis supervisor after my 5 year absence from academia. Dr. Alaca also directed my studies towards Lambert series, and particularly the product of Lambert series theorem given in [2, p. 7]. The results of this thesis could not have been achieved without his guidance.

I would like to thank Dr. Kenneth S. Williams, who made many helpful suggestions regarding my thesis. I am very grateful to Dr. Ayse Alaca for her handwritten notes of the proof of Theorem 3.1.3.

Further thanks go to all the professors at Carleton University whose courses I have enjoyed over the years. Finally, I would like to thank the staff of the School of Mathematics and Statıstics, particularly Cate Palmer, whose aid has been invaluable. 


\section{Contents}

$\begin{array}{ll}\text { Abstract } & \text { i }\end{array}$

$\begin{array}{ll}\text { Acknowledgements } & \text { ii }\end{array}$

1 Introduction $\quad 1$

2 Basic Concepts $\quad 5$

2.1 Terminology and Definitions . . . . $\quad . \quad . \quad \ldots \quad$. $\quad$. 5

2.2 Power Series and Formal Power Series . . . . . . . . . . . . . . 7

2.3 Divisor Functions . . . . . . . . . . . . . . . . . . . . . . 9

2.4 Convolution Sums . . . . . . . . . . . . . . . . . . . 10

2.5 Geometric Series . . . . . . . . . . . . . . . . . . . 11

3 Lambert Series Identities $\quad 13$

3.1 Lambert Series $\ldots \ldots \ldots \ldots$

3.2 Expressing Lambert Series As Power Series . . . . . . . . . . . . . 18

3.3 A Recursive Formula for $S_{a, b}(n) \ldots \ldots \ldots \ldots \ldots$

3.4 Other Lambert Series Products . . . . . . . . . . . . . . . . . 27

4 Convolution Sum Identities $\quad 36$

4.1 Linear Identities $\ldots \ldots \ldots$ 
4.2 Ramanujan's Approximation . . . . . . . . . . . . . . . . 38

5 New Convolution Sum Identities 44

$5.1 \quad$ New Identities . . . . . . . . . . . . . . . . . . . . . . . . . 42

5.2 Weight $14 \ldots \ldots \ldots \ldots \ldots \ldots \ldots \ldots \ldots \ldots \ldots \ldots$

5.3 Weight $16 \ldots \ldots \ldots \ldots \ldots \ldots \ldots \ldots \ldots \ldots$

5.4 Weight $18 \ldots \ldots \ldots \ldots$

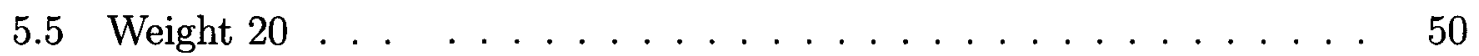

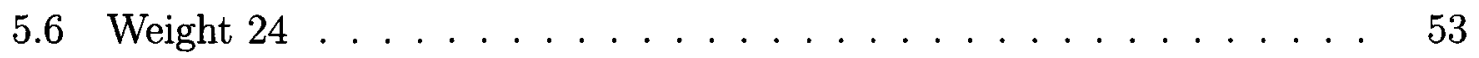

6 Further Results and Future Research Areas $\quad 58$

6.1 Another Recurrence Formula . . . . . . . . . . . . . . . . . . 58

6.2 Bernoulli's Identity . . . . . . . . . . . . . . . . . . 62

6.3 Twisted Convolution Sums . . . . . . . . . . . . . . . . . 64

6.4 A Comparison of $S_{a, b}(n)$ and $S_{a, b}^{*}(n) \ldots \ldots \ldots 6$ 


\section{Chapter 1}

\section{Introduction}

We let $\mathbb{N}, \mathbb{Z}, \mathbb{R}$ and $\mathbb{C}$ denote the sets of natural numbers, integers, real numbers and complex numbers, respectively, and we let $\mathbb{N}_{0}=\mathbb{N} \cup\{0\}$. For $n \in \mathbb{N}$ and $a \in \mathbb{N}_{0}$, the divisor function $\sigma_{a}(n)$ is defined to be the sum of the $a^{\text {th }}$ powers of the positive divisors of $n$, that is, $\sum_{d \mid n} d^{a}$. We adopt the common notation of $\sigma_{1}(n)=\sigma(n)$.

For any function $f: \mathbb{Z} \rightarrow \mathbb{C}$, a series of the form

$$
\sum_{n=1}^{\infty} f(n) \frac{x^{n}}{1-x^{n}}, \quad x \in \mathbb{C}
$$

with $|x|<1$, is called a Lambert series, see for example [4, p. 24]. Lambert series are of interest to number theory for many reasons. Our interest lies in the fact that a Lambert series may be rewritten as a series in powers of $x$, where the coefficient of any term $x^{n}$ in this power series is the sum of the function $f$ evaluated at all divisors of $n$, that is, $\sum_{d \mid n} f(d)$. We refer to this as the fundamental property of Lambert series. In 1747, Euler sent a letter to Goldbach detailing a recursive definition of the divisor function $\sigma(n)[5$, p. 40]. Euler was able to derive this recurrence relation by equating a power series with a Lambert series. We are interested in Lambert series for simlar reasons. Specifically, we are interested in the product of two Lambert series.

The word convolution indicates a twist or a coiling. In a mathematical context, a 
convolution refers to the relationship between two functions, or two similar objects. For $n \in \mathbb{N}$ and functions $f, g: \mathbb{Z} \rightarrow \mathbb{C}$, we are concerned with sums of the form $\sum_{m=1}^{n-1} f(m) g(n-m)$. We call this the convolution sum of $f$ and $g$. In this case, we can see that the convolution refers to the relationship between the values of the two functions, and does not indicate anything about the functions themselves.

For our purposes, we are interested in convolution sums involving divisor functions, that is, sums of the form $\sum_{m=1}^{n-1} \sigma_{a}(m) \sigma_{b}(n-m)$. Specifically, we will show that, for all odd positive integers $a$ and $b$, there exists a recurrence relation for the convolution $\operatorname{sum} \sum_{m=1}^{n-1} \sigma_{a}(m) \sigma_{b}(n-m)$. As these sums are of primary interest, when speaking of convolution sums, it is understood that the functions in question are divisor functions.

The first known instance of a value for these convolution sums came in 1862, when Besge sent a letter to Liouville giving the value of $\sum_{m=1}^{n-1} \sigma(m) \sigma(n-m)$ [7]. It is believed by some that Besge is a pseudonym for Liouville [20, p. 81]. In fact, Besge's value for $\sum_{m=1}^{n-1} \sigma(m) \sigma(n-m)$ can be derived from an identity of Liouville [26, p. 141]. We will operate under the assumption that it was Liouville's arithmetic identities that were the catalyst for investıgating $\sum_{m=1}^{n-1} \sigma(m) \sigma(n-m)$.

In 1916, Ramanujan gave an expression for $\sum_{m=0}^{n} \sigma_{a}(m) \sigma_{b}(n-m)$, for all odd positive integers $a$ and $b$. His definition differs from our definition given above, in that he defines $\sigma_{a}(0)$ to be $\frac{1}{2} \zeta(-a)$, where $\zeta(s)$ is the Riemann zeta function. However, Ramanujan's expression was not explicit for all odd values of $a$ and $b$, and indeed, Ramanujan's expression involved a big-Oh error term. He was able to show that for certain pairs of odd positive integers $a$ and $b$, the convolution sum $\sum_{m=1}^{n-1} \sigma_{a}(m) \sigma_{b}(n-m)$ can be expressed as a linear combination of divisor functions. It is notable that Ramanujan used Lambert series throughout his paper [22], [16, pp. 136-162].

Earlier investigations into these convolution sums were undertaken by Glaisher 
$[10],[11],[12]$, who investigated convolution sums of many functions involving sums of divisors. Later investigations were conducted by Lahiri [18], [19], who gave many congruence identities for these convolution sums.

In modern research involving Eisenstein series or modular forms, it often occurs that the values for certain convolution sums are used. The value of $\sum_{m=1}^{n-1} \sigma(m) \sigma(n-m)$ appears in the work of Skoruppa [25] and Royer [23], who were investrgating Eisenstein series, and quasimodular forms, respectively. Some of Lahiri's identities are discussed by Berndt and Yee [6], in their investigation of congruences of quotients of Eisenstein series. Hahn [15] used Eisenstein series in order to study convolution sums similar to those studied by Glaisher.

Recently, Huard, Ou, Spearman and Williams [17], by elementary methods, were able to give a generalization of an arithmetic identity of Liouville. It was this identity of Liouville that was first used by Besge to evaluate $\sum_{m=1}^{n-1} \sigma(m) \sigma(n-m)[26]$. The generalization of Huard, Ou, Spearman and Williams was used to great effect to evaluate those convolution sums $\sum_{m=1}^{n-1} \sigma_{a}(m) \sigma_{b}(n-m)$ which are expressible as linear combinations of the divisor functions. It is notable that these values were achieved through elementary arithmetic methods. Our recurrence relation is also derived in a basically elementary manner as well, although we use a few ideas from the theory of complex variables.

We begin by giving a brief overview of the basic material needed in Chapter 2 . The next chapter introduces various Lambert series identities. In this chapter, we make great use of a theorem of Alaca, Alaca, McAfee and Williams, proved in [2, pp 25-27], to derive the main result. Subsequent chapters are concerned with evaluating the convolution sums $\sum_{m=1}^{n-1} \sigma_{a}(m) \sigma_{b}(n-m)$ for various $a$ and $b$. In Chapter 4 we show our formula reproduces known results. In Chapter 5, we use our formula to give a series of new convolution sum identities. By way of conclusion, we discuss the 
strengths and weaknesses of our formula compared with Ramanujan's formula. We are also able to relate our formula with a recurrence relation given in [26, p. 143]. Additionally, we briefly mention how our method may be used in the evaluation of certain twrsted convolution sums of the form $\sum_{m<n / k} \sigma_{a}(n-k m) \sigma_{b}(m)$. 


\section{Chapter 2}

\section{Basic Concepts}

\subsection{Terminology and Definitions}

We review some of the basic mathematical tools and definitions we will be using throughout.

Definition 2.1.1. Any function $f: \mathbb{N} \rightarrow \mathbb{C}$ is called an arthmetıc, or arıthmetıcal function.

Definition 2.1.2. Suppose $f$ has the domain $D \subseteq \mathbb{R}$ and $f: D \rightarrow \mathbb{R}$. If, for all $x \in D$, we have $-f(x)=f(-x)$, then $f$ is said to be an odd function. Instead, if for

all $x \in D$ we have that $f(x)=f(-x)$, then $f$ is said to be an even function. Note that $f(0)=0$ when $f$ is odd.

Definition 2.1.3. For $0<x<\infty$, we define the Gamma function, denoted $\Gamma(x)$, as follows:

$$
\Gamma(x)=\int_{0}^{\infty} t^{x-1} e^{-t} d t .
$$

For any $n \in \mathbb{N}$, integration by parts of the Gamma function yields

$$
\Gamma(n+1)=n !,
$$


see for example $[24, \mathrm{p}$ 192].

Definition 2.1.4. Let $z \in \mathbb{C}$ satisfy $|z|<2 \pi$. Then the Bernoulli numbers $B_{n}$ are given by the infinite series

$$
\frac{z}{e^{z}-1}=\sum_{n=0}^{\infty} \frac{B_{n}}{n !} z^{n}
$$

see for example [3, pp. 264-265].

We note that $B_{0}=1$ and $B_{1}=-\frac{1}{2}$. For $n \geq 2$ we have the recurrence relation

$$
B_{n}=\sum_{k=0}^{n}\left(\begin{array}{l}
n \\
k
\end{array}\right) B_{k}
$$

Definition 2.1.5. For $s \in \mathbb{C}$ with $\Re(s)>1$, we define the Riemann zeta function, denoted $\zeta(s)$, by the series

$$
\zeta(s)=\sum_{n=1}^{\infty} \frac{1}{n^{s}}
$$

The analytic continuation of this function to the whole complex plane, given by the functional equation

$$
\zeta(s)=2 \Gamma(1-s)(2 \pi)^{s-1} \sin \left(\frac{s \pi}{2}\right) \zeta(1-s),
$$

provides the definition of $\zeta(s)$ for $s \in \mathbb{C}$. The Riemann zeta function is analytic everywhere in $\mathbb{C}$ except for a simple pole at $s=1$ with residue 1 , see for example $[9$, pp. 10-13]. 
When we evaluate $\zeta(s)$ for even positive integers, we find

$$
\zeta(2 n)=\frac{(2 \pi)^{2 n}(-1)^{n+1} B_{2 n}}{2 \cdot(2 n) !}
$$

By evaluating $\zeta(s)$ for negative integers, we obtain

$$
\zeta(-n)=(-1)^{n} \frac{B_{n+1}}{n+1}
$$

When we evaluate the Riemann zeta function at 0 , we have

$$
\zeta(0)=B_{1}=-\frac{1}{2}
$$

see for example [9, p. 12]

Definition 2.1.6. Ramanujan's tau function, an arithmetical function, denoted $\tau(n)$, is defined by

$$
q \prod_{n=1}^{\infty}\left(1-q^{n}\right)^{24}=\sum_{n=1}^{\infty} \tau(n) q^{n}
$$

for $q \in \mathbb{C}$, with $|q|<1$, see [16, p. 151].

\subsection{Power Series and Formal Power Series}

Definition 2.2.1. A series of the form

$$
\sum_{n=0}^{\infty} c(n) x^{n}
$$

is called a power series in $x$. The values $c(n)$ are called the coefficients of the series and both $c(n)$ and $x$ may be real or complex numbers. For each power series, there 
exists a corresponding radius of convergence $R \geq 0$. If we let $R$ denote the radius of convergence of the power series given by (2.2.1), then this power series converges absolutely if $|x|<R$, and diverges if $|x|>R$, see for example, [1, p. 38].

A formal power series is one in which we are not concerned with matters of convergence or divergence, and so we do not assign a numerical value to $x$. A formal power series is still given by $\sum_{n=0}^{\infty} c(n) x^{n}$, but we are only interested in the sequence of coefficients

$$
c(0), c(1), \ldots, c(n), \ldots,
$$

and we use the $x^{n}$ term solely to locate the $n^{\text {th }}$ coefficient $c(n)$. With this structure, we may operate on formal power series as if they were convergent power series. We note that any convergent power series may be considered a formal power series.

Definition 2.2.2. Let $A(x)$ and $B(x)$ be two formal power series, and suppose

$$
A(x)=\sum_{n=0}^{\infty} a(n) x^{n}, \text { and } B(x)=\sum_{n=0}^{\infty} b(n) x^{n} .
$$

Then we say $A(x)=B(x)$ if and only if $a(n)=b(n)$ for all $n \geq 0$. We define the sum and product of formal power series as follows:

$$
\begin{aligned}
A(x)+B(x) & =\sum_{n=0}^{\infty}(a(n)+b(n)) x^{n}, \\
A(x) B(x) & =\sum_{n=0}^{\infty}\left(\sum_{m=0}^{n} a(m) b(n-m)\right) x^{n} .
\end{aligned}
$$

The bracketed term in the product $A(x) B(x)$ is called the Cauchy product of the sequences $(a(n))$ and $(b(n))$, see for example [3, pp. 41-42]. 
Suppose now we define the following formal power series,

$$
A(x)=\sum_{n=1}^{\infty} a(n) x^{n} \text { and } B(x)=\sum_{n=1}^{\infty} b(n) x^{n}
$$

By taking the lower bound of each summation to be 1 instead of 0 , we must remove those terms from our original Cauchy product which contain either $a(0)$ or $b(0)$. The Cauchy product of $(a(n))$ and $(b(n))$ then becomes

$$
\sum_{m=1}^{n-1} a(m) b(n-m)
$$

When $n=1$ this sum is empty and has the value 0 . In general, for $k \in \mathbb{N}$, we have

$$
\sum_{n=k}^{\infty} a(n) x^{n} \sum_{n=k}^{\infty} b(n) x^{n}=\sum_{n=k}^{\infty}\left(\sum_{m=k}^{n-k} a(m) b(n-m)\right) x^{n}
$$

\subsection{Divisor Functions}

Definition 2.3.1. For $n \in \mathbb{N}$ and $a \in \mathbb{N}_{0}$ we defined the divısor function $\sigma_{a}(n)$ in Chapter 1 by

$$
\sigma_{a}(n)=\sum_{d \mid n} d^{a}
$$

and we denote $\sigma_{1}(n)$ by $\sigma(n)$. 


\subsection{Convolution Sums}

Definition 2.4.1. For any function $f: \mathbb{Z} \rightarrow \mathbb{C}$ and for $n \in \mathbb{N}$ we say the sum

$$
\sum_{m=1}^{n-1} f(m) f(n-m)
$$

is a convolution sum of the function $f$.

By changing the summation variable from $m$ to $n-m$, it is plain to see that

$$
\sum_{m=1}^{n-1} f(m) f(n-m)=\sum_{m=1}^{n-1} f(n-m) f(m) .
$$

We are more interested in those sums resulting from a convolution between two different functions.

Definition 2.4.2. For any functions $f, g: \mathbb{Z} \rightarrow \mathbb{C}$, and for $n \in \mathbb{N}$ we define the convolution sum of $f$ and $g$ to be

$$
\sum_{m=1}^{n-1} f(m) g(n-m)=\sum_{m=1}^{n-1} f(n-m) g(m) .
$$

Definition 2.4.3. For $a, b \in \mathbb{N}$, we define $S_{a, b}: \mathbb{N} \rightarrow \mathbb{Z}$ by

$$
S_{a, b}(n):=\sum_{m=1}^{n-1} \sigma_{a}(m) \sigma_{b}(n-m)
$$

When $n=1$, this gives the empty sum, and hence $S_{a, b}(1)=0$. So we usually assume $n \geq 2$ when referring to this convolution sum.

We will have occasion to consider what happens when we evaluate the divisor sum at zero. This will occur when we concern ourselves with Ramanujan's approximation of $S_{a, b}(n)$. Thus, we refer to [16, p. 136] for the following definition. 
Definition 2.4.4. For any odd positive integer $a$, we define

$$
\sigma_{a}(0)=\frac{1}{2} \zeta(-a)=-\frac{1}{2} \frac{B_{a+1}}{a+1}
$$

where $B_{n}$ is the $n^{\text {th }}$ Bernoulli number and $\zeta(s)$ is the Riemann zeta function. Note that we have tacitly used property (2.1.3) in (2.4.2). With this, we define Ramanujan's convolution sum $S_{a, b}^{*}: \mathbb{N}_{0} \rightarrow \mathbb{Z}$ by

$$
S_{a, b}^{*}(n):=\sum_{m=0}^{n} \sigma_{a}(m) \sigma_{b}(n-m)
$$

\subsection{Geometric Series}

Suppose $q \in \mathbb{R}$ or $\mathbb{C}$ and satısfies $|q|<1$ Consıder first the finite sum

$$
1+q+q^{2}+q^{3}+\cdots+q^{n}=\sum_{r=0}^{n} q^{r}
$$

Multiplying this sum by $(1-q)$, we find

$$
(1-q) \cdot\left(1+q+q^{2}+\cdots+q^{n}\right)=1+(q-q)+\left(q^{2}-q^{2}\right)+\cdots+\left(q^{n}-q^{n}\right)-q^{n+1} .
$$

This means

$$
\left(\sum_{r=0}^{n} q^{r}\right) \cdot(1-q)=1-q^{n+1} \text { or } \sum_{r=0}^{n} q^{r}=\frac{1-q^{n+1}}{1-q}
$$

Hence,

$$
\sum_{r=0}^{\infty} q^{r}=\lim _{n \rightarrow \infty} \sum_{r=0}^{n} q^{r}=\lim _{n \rightarrow \infty} \frac{1-q^{n+1}}{1-q}=\frac{1}{1-q}
$$


as $\lim _{n \rightarrow \infty} q^{n+1}=0$ for $|q|<1$. Differentiating both sides of (2.5.1) with respect to $q$ we obtain

$$
\sum_{r=1}^{\infty} r q^{r-1}=\frac{1}{(1-q)^{2}}
$$

Thus

$$
\sum_{r=1}^{\infty}(r-1) q^{r}=\frac{q}{(1-q)^{2}}-\left(\frac{1}{1-q}-1\right)=\frac{q^{2}}{(1-q)^{2}}
$$




\section{Chapter 3}

\section{Lambert Series Identities}

\subsection{Lambert Series}

Let $q \in \mathbb{C}$ satisfy $|q|<1$ and for $n \in \mathbb{N}$ we let

$$
u_{n}=\frac{q^{n}}{1-q^{n}} .
$$

From (2.5.1), we deduce

$$
u_{n}=q^{n} \cdot \sum_{k=0}^{\infty}\left(q^{n}\right)^{k}=\sum_{k=1}^{\infty} q^{n k}
$$

The following theorem is the fundamental reason why we can equate Lambert series with power series.

Theorem 3.1.1. For any functıon $f: \mathbb{Z} \rightarrow \mathbb{C}$ we have

$$
\sum_{n=1}^{\infty} f(n) u_{n}=\sum_{n=1}^{\infty} q^{n} \sum_{d \mid n} f(d)
$$


Proof. We have

$$
\sum_{n=1}^{\infty} f(n) u_{n}=\sum_{n=1}^{\infty} f(n) \sum_{k=1}^{\infty} q^{n k}=\sum_{n, k \geq 1} f(n) q^{n k}=\sum_{\substack { N=1 \\
\begin{subarray}{c}{n, k \geq 1 \\
n k=N{ N = 1 \\
\begin{subarray} { c } { n , k \geq 1 \\
n k = N } }\end{subarray}}^{\infty} f(n) q^{N}=\sum_{N=1}^{\infty} \sum_{n \mid N} f(n) q^{N}
$$

completing the proof.

We immediately deduce from Theorem 3.1.1 that

$$
\sum_{n=1}^{\infty} n^{a} u_{n}=\sum_{n=1}^{\infty} q^{n} \sigma_{a}(n)
$$

Although the following is not strictly given as a Lambert series, we will make use of it later on.

Theorem 3.1.2. For any functions $f, g: \mathbb{Z} \rightarrow \mathbb{C}$ we have

$$
\sum_{n=1}^{\infty} f(n) \sum_{k=1}^{\infty} g(k) q^{n k}=\sum_{n=1}^{\infty} q^{n} \sum_{d \mid n} f(d) g\left(\frac{n}{d}\right)
$$

Proof. We have

$$
\begin{aligned}
\sum_{n=1}^{\infty} f(n) \sum_{k=1}^{\infty} g(k) q^{n k}=\sum_{n, k \geq 1} f(n) g(k) q^{n k} & =\sum_{\substack { N=1 \\
\begin{subarray}{c}{n, k \geq 1 \\
n k=N{ N = 1 \\
\begin{subarray} { c } { n , k \geq 1 \\
n k = N } }\end{subarray}} f(n) g(k) q^{N} \\
& =\sum_{N=1}^{\infty} \sum_{n \mid N} f(n) g\left(\frac{N}{n}\right) q^{N} .
\end{aligned}
$$

The following theorem is due to Alaca, Alaca, McAfee and Williams [2, p. 7].

Theorem 3.1.3. If $f, g: \mathbb{Z} \rightarrow \mathbb{C}$ are odd functıons satısfyıng $|f(n)| \leq A n^{r},|g(n)| \leq$ 
$B n^{s}$ for some $A>0, B>0, r \geq 0, s \geq 0$ then

$$
\left(\sum_{n=1}^{\infty} u_{n} f(n) \cos n \theta\right)\left(\sum_{n=1}^{\infty} u_{n} g(n) \cos n \theta\right)=\frac{1}{2} \sum_{n=1}^{\infty} f(n) g(n) u_{n}^{2}+\frac{1}{2} \sum_{n=1}^{\infty} c_{n} \cos n \theta
$$

where, for $n \in \mathbb{N}$,

$$
\begin{aligned}
c_{n}=u_{n} & \sum_{m=1}^{\infty}[f(m+n) g(m)+g(m+n) f(m)] u_{m} \\
& +u_{n} \sum_{m=1}^{\infty}[f(m) g(n-m)+f(n-m) g(m)] u_{m} \\
& +\sum_{m=n+1}^{\infty}[f(m) g(n-m)+f(n-m) g(m)] u_{m} \\
& +u_{n} \sum_{m=1}^{n-1} f(m) g(n-m) .
\end{aligned}
$$

Proof. We emulate the proof given in [2, pp. 25-27]. First, it is given in [2, p. 22] that for $k, n \in \mathbb{N}$ we have

$$
u_{n} u_{k+n}=u_{k}\left(u_{n}-u_{k+n}\right)-u_{k+n}
$$

and

$$
u_{n} u_{k-n}=u_{k}\left(1+u_{n}+u_{k-n}\right) \text { for } n<k \text {. }
$$

Next, we have that

$$
\left(\sum_{m=1}^{\infty} f(m) \cos m \theta u_{m}\right)\left(\sum_{n=1}^{\infty} g(n) \cos n \theta u_{n}\right)
$$




$$
\begin{aligned}
& =\sum_{m, n=1}^{\infty} f(m) g(n) \cos m \theta \cos n \theta u_{m} u_{n} \\
& =\frac{1}{2} \sum_{m, n=1}^{\infty} f(m) g(n)[\cos (m-n) \theta+\cos (m+n) \theta] u_{m} u_{n}=\frac{1}{2} \sum_{k=0}^{\infty} c_{k} \cos k \theta
\end{aligned}
$$

where

$$
c_{0}=\sum_{\substack{m, n=1 \\ m=n}}^{\infty} f(m) g(n) u_{m} u_{n}=\sum_{n=1}^{\infty} f(n) g(n) u_{n}^{2}
$$

and for $k \in \mathbb{N}, c_{k}$ is given by

$$
\begin{aligned}
c_{k} & =\sum_{m+n=k} f(m) g(n) u_{m} u_{n}+\sum_{m-n=k} f(m) g(n) u_{m} u_{n}+\sum_{m-n=-k} f(m) g(n) u_{m} u_{n} \\
& =\sum_{n=1}^{k-1} f(k-n) g(n) u_{n} u_{k-n}+\sum_{n=1}^{\infty} f(k+n) g(n) u_{n} u_{k+n}+\sum_{m=1}^{\infty} f(m) g(k+m) u_{m} u_{k+m} \\
& =\sum_{n=1}^{k-1} f(k-n) g(n) u_{n} u_{k-n}+\sum_{n=1}^{\infty}[f(k+n) g(n)+f(n) g(k+n)] u_{n} u_{k+n} .
\end{aligned}
$$

Set

$$
C(k, n)=f(k+n) g(n)+f(n) g(k+n),
$$

which means

$$
\begin{aligned}
C(k, n-k)=f(n) g(n-k)+f(n-k) g(n) & =-(f(n) g(k-n)+f(k-n) g(n)) \\
& =-C(k, k-n) .
\end{aligned}
$$

As $f$ and $g$ are odd functions, we have $C(k, 0)=f(k) g(0)+f(0) g(k)=0$. Using 
(3.1.5) and (3.1.6) we arrive at

$$
\begin{aligned}
c_{k}= & \sum_{n=1}^{k-1} f(k-n) g(n) u_{k}\left(1+u_{n}+u_{k-n}\right)+u_{k} \sum_{n=1}^{\infty} C(k, n) u_{n}-u_{k} \sum_{n=1}^{\infty} C(k, n) u_{k+n} \\
& -\sum_{n=1}^{\infty} C(k, n) u_{k+n} \\
= & \sum_{n=1}^{k-1} f(k-n) g(n) u_{k}\left(1+u_{n}+u_{k-n}\right)+u_{k} \sum_{n=1}^{k-1} C(k, n) u_{n}+u_{k}^{2} C(k, k) \\
& +u_{k} \sum_{n=k+1}^{\infty} C(k, n) u_{n}-u_{k} \sum_{n=1}^{\infty} C(k, n) u_{k+n}-\sum_{n=1}^{\infty} C(k, n) u_{k+n}
\end{aligned}
$$

We replace $n$ by $n-k$ in the last two sums of (3.1.7) to obtain

$$
\begin{aligned}
c_{k}= & \sum_{n=1}^{k-1} f(k-n) g(n) u_{k}\left(1+u_{n}+u_{k-n}\right)+u_{k} \sum_{n=1}^{k-1} C(k, n) u_{n}+u_{k}^{2} C(k, k) \\
& +u_{k} \sum_{n=k+1}^{\infty} C(k, n) u_{n}-u_{k} \sum_{n=k+1}^{\infty} C(k, n-k) u_{n}-\sum_{n=k+1}^{\infty} C(k, n-k) u_{n} \\
= & u_{k} \sum_{n=1}^{k-1} f(k-n) g(n)+u_{k} \sum_{n=1}^{k-1} f(k-n) g(n) u_{n}+u_{k} \sum_{n=1}^{k-1} f(n) g(k-n) u_{n} \\
& +u_{k} \sum_{n=1}^{k-1} C(k, n) u_{n}+u_{k}^{2} C(k, k)+u_{k} \sum_{n=k+1}^{\infty} C(k, n) u_{n}+u_{k} \sum_{n=k+1}^{\infty} C(k, k-n) u_{n} \\
& +\sum_{n=k+1}^{\infty} C(k, k-n) u_{n}
\end{aligned}
$$

Finally, we group together similar terms to conclude

$$
\begin{aligned}
c_{k}= & u_{k} \sum_{n=1}^{k-1} f(n)[g(k+n)+g(k-n)] u_{n}+u_{k} \sum_{n=1}^{k-1} g(n)[f(k+n)+f(k-n)] u_{n} \\
& +u_{k} \sum_{n=k+1}^{\infty} f(n)[g(k+n)+g(k-n)] u_{n}+u_{k} \sum_{n=k+1}^{\infty} g(n)[f(k+n)+f(k-n)] u_{n} \\
& +u_{k}^{2} C(k, k)+u_{k} \sum_{n=1}^{k-1} f(k-n) g(n)+\sum_{n=k+1}^{\infty} C(k, k-n) u_{n}
\end{aligned}
$$




$$
\begin{aligned}
= & u_{k} \sum_{n=1}^{\infty} f(n)[g(k+n)+g(k-n)] u_{n}-u_{k}^{2} g(k)(f(2 k)-f(0)) \\
& +u_{k} \sum_{n=1}^{\infty} f(n)[g(k+n)+g(k-n)] u_{n}-u_{k}^{2} f(k)(g(2 k)-g(0)) \\
& +u_{k}^{2}(f(2 k) g(k)+f(k) g(2 k))+u_{k} \sum_{n=1}^{k-1} f(n) g(k-n)+\sum_{n=k+1}^{\infty} C(k, k-n) u_{n} \\
= & u_{k} \sum_{n=1}^{\infty}[f(n) g(k+n)+f(k+n) g(n)] u_{n}+u_{k} \sum_{n=1}^{\infty}[f(n) g(k-n)+f(k-n) g(n)] u_{n} \\
& +\sum_{n=k+1}^{\infty}[f(n) g(k-n)+f(k-n) g(n)] u_{n}+u_{k} \sum_{n=1}^{k-1} f(n) g(k-n),
\end{aligned}
$$

completing the proof of the theorem.

\subsection{Expressing Lambert Series As Power Series}

By (3.1.2), for any positive integers $a$ and $b$, we have

$$
\left(\sum_{n=1}^{\infty} n^{a} u_{n}\right)\left(\sum_{n=1}^{\infty} n^{b} u_{n}\right)=\left(\sum_{n=1}^{\infty} q^{n} \sigma_{a}(n)\right)\left(\sum_{n=1}^{\infty} q^{n} \sigma_{b}(n)\right)
$$

and the right hand side of (3.2.1) may be written as a formal power series, namely,

$$
\sum_{n=1}^{\infty} q^{n}\left(\sum_{m=1}^{n-1} \sigma_{a}(m) \sigma_{b}(n-m)\right)=\sum_{n=1}^{\infty} q^{n} S_{a, b}(n)
$$

The left hand side of (3.21) can be drawn from Theorem 3.1.3, under the conditions that $\theta=0, f(n)=n^{a}, g(n)=n^{b}$ for arbitrary odd positive integers $a$ and $b$. Our aim now is to express each term of of the right hand side of (3.1.3) as a formal power series. 
Lemma 3.2.1. For any functions $f, g: \mathbb{Z} \rightarrow \mathbb{C}$, we have

$$
\sum_{n=1}^{\infty} f(n) g(n) u_{n}^{2}=\sum_{n=1}^{\infty} q^{n} \sum_{d \mid n}\left(\frac{n}{d}-1\right) f(d) g(d)
$$

Proof. First, notice by $(25.3)$ that $u_{n}^{2}=\sum_{k=1}^{\infty}(k-1) q^{n k}$. Thus

$$
\begin{aligned}
\sum_{n=1}^{\infty} f(n) g(n) u_{n}^{2} & =\sum_{n=1}^{\infty} f(n) g(n) \sum_{k=1}^{\infty}(k-1) q^{n k} \\
& =\sum_{n, k \geq 1} f(n) g(n)(k-1) q^{n k} \\
& =\sum_{N=1}^{\infty}\left(\sum_{\substack{n, k \geq 1 \\
n k=N}} f(n) g(n)(k-1)\right) q^{N} \\
& =\sum_{N=1}^{\infty}\left(\sum_{n \mid N}\left(\frac{N}{n}-1\right) f(n) g(n)\right) q^{N}
\end{aligned}
$$

as asserted.

Lemma 3.2.2. For any functions $f, g: \mathbb{Z} \rightarrow \mathbb{C}$, we have

$$
\begin{aligned}
& \sum_{n=1}^{\infty} u_{n} \cos n \theta \sum_{m=1}^{\infty} f(n+m) g(m) u_{m}=\sum_{n=1}^{\infty} q^{n} \sum_{m=1}^{\infty} q^{m} \sum_{d^{\prime} \mid n} \cos d^{\prime} \theta \sum_{d \mid m} f\left(d^{\prime}+d\right) g(d) \\
& \sum_{n=1}^{\infty} u_{n} \cos n \theta \sum_{m=1}^{\infty} f(n-m) g(m) u_{m}=\sum_{n=1}^{\infty} q^{n} \sum_{m=1}^{\infty} q^{m} \sum_{d^{\prime} \mid n} \cos d^{\prime} \theta \sum_{d \mid m} f\left(d^{\prime}-d\right) g(d)
\end{aligned}
$$

Proof. We consider $\sum_{n=1}^{\infty} u_{n} \cos n \theta \sum_{m} u_{m} f(n \pm m) g(m)$. We have

$$
\sum_{n=1}^{\infty} u_{n} \cos n \theta \sum_{m=1}^{\infty} f(n \pm m) g(m) u_{m}=\sum_{d^{\prime}=1}^{\infty} u_{d^{\prime}} \cos d^{\prime} \theta \sum_{d=1}^{\infty} f\left(d^{\prime} \pm d\right) g(d) u_{d}
$$




$$
\begin{aligned}
= & \sum_{d^{\prime}=1}^{\infty} u_{d^{\prime}} \cos d^{\prime} \theta \sum_{d=1}^{\infty} f\left(d^{\prime} \pm d\right) g(d) \sum_{t=1}^{\infty} q^{d t} \\
= & \sum_{d^{\prime}=1}^{\infty} u_{d^{\prime}} \cos d^{\prime} \theta \sum_{n=1}^{\infty} \sum_{\substack{d, t \geq 1 \\
d t=n}} f\left(d^{\prime} \pm d\right) g(d) q^{n} \\
= & \sum_{n=1}^{\infty} q^{n} \sum_{d^{\prime}=1}^{\infty} u_{d^{\prime}} \cos d^{\prime} \theta \sum_{d \mid n} f\left(d^{\prime} \pm d\right) g(d) \\
= & \sum_{n=1}^{\infty} q^{n} \sum_{d^{\prime}=1}^{\infty} \cos d^{\prime} \theta \sum_{u=1}^{\infty} q^{d^{\prime} u} \sum_{d \mid n} f\left(d^{\prime} \pm d\right) g(d) \\
= & \sum_{n=1}^{\infty} q^{n} \sum_{m=1}^{\infty} q^{m} \sum_{\substack{d^{\prime}, u \geq 1 \\
d^{\prime} u=m}} \cos d^{\prime} \theta \sum_{d \mid n} f\left(d^{\prime} \pm d\right) g(d) \\
= & \sum_{n=1}^{\infty} q^{n} \sum_{m=1}^{\infty} q^{m} \sum_{d^{\prime} \mid m} \cos d^{\prime} \theta \sum_{d \mid n} f\left(d^{\prime} \pm d\right) g(d),
\end{aligned}
$$

as asserted.

Lemma 3.2.3. For any functions $f, g: \mathbb{Z} \rightarrow \mathbb{C}$, we have

$$
\sum_{n=1}^{\infty} \cos n \theta \sum_{m=n+1}^{\infty} f(n-m) g(m) u_{m}=\sum_{n=1}^{\infty} q^{n} \sum_{d \mid n} g(d) \sum_{m=1}^{d-1} f(-m) \cos (d-m) \theta
$$

Proof. We have

$$
\begin{aligned}
\sum_{n=1}^{\infty} \cos n \theta \sum_{m=n+1}^{\infty} f(n-m) g(m) u_{m} & =\sum_{n=1}^{\infty} \cos n \theta \sum_{m=n+1}^{\infty} f(n-m) g(m) \sum_{t=1}^{\infty} q^{m t} \\
& =\sum_{k=1}^{\infty} q^{k} \sum_{n=1}^{\infty} \sum_{m=n+1}^{\infty} \cos n \theta f(n-m) g(m) \\
& =\sum_{k=1}^{\infty} q^{k} \sum_{m=1}^{\infty} \sum_{n=1}^{m-1} \cos n \theta f(n-m) g(m) \\
& =\sum_{k=1}^{\infty} q^{k} \sum_{m \mid k} g(m) \sum_{n=1}^{m-1} \cos (m-n) \theta f(-n)
\end{aligned}
$$




$$
=\sum_{n=1}^{\infty} q^{n} \sum_{d \mid n} g(d) \sum_{m=1}^{d-1} f(-m) \cos (d-m) \theta
$$

as claimed.

Lemma 3.2.4. For any functions $f, g: \mathbb{Z} \rightarrow \mathbb{C}$, we have

$$
\sum_{n=1}^{\infty} u_{n} \cos n \theta \sum_{m=1}^{n-1} f(m) g(n-m)=\sum_{n=1}^{\infty} q^{n} \sum_{d \mid n} \cos d \theta \sum_{m=1}^{d-1} f(m) g(d-m)
$$

Proof. We write

$$
\phi(n)=\sum_{m=1}^{n-1} f(m) g(n-m)
$$

and then

$$
\sum_{n=1}^{\infty} u_{n} \cos n \theta \phi(n)=\sum_{n=1}^{\infty} q^{n} \sum_{d \mid n} \cos d \theta \phi(d)
$$

by Theorem 3.1.1. Replacing $\phi(d)$ by $\sum_{m=1}^{n-1} f(m) g(d-n)$ we obtain the assertion of the lemma.

We are no longer interested in any cosine terms, so we fix $\theta=0$. Using Lemmas 3.2.1 - 3.2.4 and Theorem 3.1.3, we deduce the following theorem.

Theorem 3.2.5. For odd functıons $f, g: \mathbb{Z} \rightarrow \mathbb{C}$, we have

$$
\begin{aligned}
2\left(\sum_{n=1}^{\infty} f(n) u_{n}\right) & \left(\sum_{m=1}^{\infty} g(m) u_{m}\right) \\
& =\sum_{n=1}^{\infty} q^{n} \sum_{d \mid n}\left(\frac{n}{d}-1\right) f(d) g(d)
\end{aligned}
$$




$$
\begin{aligned}
& +\sum_{n=1}^{\infty} q^{n} \sum_{m=1}^{\infty} q^{m} \sum_{d^{\prime} \mid n} \sum_{d \mid m}\left\{f\left(d^{\prime}+d\right) g(d)+g\left(d^{\prime}+d\right) f(d)\right. \\
& \left.+f\left(d^{\prime}-d\right) g(d)+g\left(d^{\prime}-d\right) f(d)\right\} \\
& -\sum_{n=1}^{\infty} q^{n}\left(\sum_{d \mid n} g(d) \sum_{m=1}^{d-1} f(m)+\sum_{d \mid n} f(d) \sum_{m=1}^{d-1} g(m)\right) \\
& +\sum_{n=1}^{\infty} q^{n} \sum_{d \mid n} \sum_{m=1}^{d-1} f(m) g(d-m) .
\end{aligned}
$$

We can see now that if we can write the second term of the right hand side of (3.2.8) as a (formal) power series, we may rewrite the entire expression as a (formal) power series. Thus, by equating coefficients, we can deduce a formula for the convolution $\operatorname{sum} \sum_{m=1}^{n-1}\left(\sum_{d \mid m} f(d)\right)\left(\sum_{d \mid n-m} g(d)\right)$.

Consider now the sum

$$
\sum_{n=1}^{\infty} q^{n} \sum_{s x+t y=n} f(s) g(t)
$$

where this inner sum is over all positive integers $s, t, x, y$ satisfying $s x+t y=n$. (We follow [17, p. 2] in this definition.) Observe that if $s x=m$ for $1 \leq m \leq n-1$, then we must have $t y=n-m$. Thus, we may rewrite (3.2.9) as

$$
\sum_{n=1}^{\infty} q^{n} \sum_{m=1}^{n-1} \sum_{s x=m} f(s) \sum_{t y-n-m} g(t)
$$

which is equivalent to

$$
\sum_{n=1}^{\infty} q^{n} \sum_{m=1}^{n-1}\left(\sum_{d \mid m} f(d)\right)\left(\sum_{d \mid n-m} g(d)\right) .
$$


Next, consider the expression

$$
\sum_{n=1}^{\infty} q^{n} \sum_{m=1}^{\infty} q^{m} \sum_{d^{\prime} \mid n} \sum_{d \mid m} f\left(d^{\prime}+d\right) g(d)
$$

We may rewrite this as

$$
\begin{aligned}
\sum_{m, n \geq 1} q^{m+n} \sum_{d^{\prime} e^{\prime}=n} \sum_{d e=m} f\left(d^{\prime}+d\right) g(d) & =\sum_{k=1}^{\infty} q^{k} \sum_{\substack{m, n \geq 1 \\
m+n=k}} \sum_{d^{\prime} e^{\prime}=n} \sum_{d e=m} f\left(d^{\prime}+d\right) g(d) \\
& =\sum_{k=1}^{\infty} q^{k} \sum_{\substack{m, n \geq 1 \\
m+n=k}} \sum_{d^{\prime} e^{\prime}+d e=m+n} f\left(d^{\prime}+d\right) g(d) \\
& =\sum_{k=1}^{\infty} q^{k} \sum_{d^{\prime} e^{\prime}+d e=k} f\left(d^{\prime}+d\right) g(d) .
\end{aligned}
$$

By relabelling indices as necessary, we deduce that

$$
\sum_{n=1}^{\infty} q^{n} \sum_{m=1}^{\infty} q^{m} \sum_{d^{\prime} \mid n} \sum_{d \mid m} f\left(d^{\prime}+d\right) g(d)=\sum_{n=1}^{\infty} q^{n} \sum_{s x+t y=n} f(t+s) g(s) .
$$

Hence, (3.2.8) may be considered as an equality between two formal power series. By equating coefficients of these formal power series, we conclude the following theorem.

Theorem 3.2.6. Let $f, g: \mathbb{Z} \rightarrow \mathbb{C}$ be odd functions. Then for all $n \in \mathbb{N}$ we have

$$
\begin{aligned}
2 \sum_{s x+t y=n} f(s) g(t)= & \sum_{d \mid n}\left(\frac{n}{d}-1\right) f(d) g(d)+\sum_{s x+t y=n}\{f(s+t) g(s)+g(s+t) f(s) \\
& +f(t-s) g(s)+g(t-s) f(s)\}-\sum_{d \mid n} g(d) \sum_{m=1}^{d-1} f(m) \\
& -\sum_{d \mid n} f(d) \sum_{m=1}^{d-1} g(m)+\sum_{d \mid n} \sum_{m=1}^{d-1} f(m) g(d-m)
\end{aligned}
$$




\subsection{A Recursive Formula for $S_{a, b}(n)$}

In this section, we present our main result. By substituting in appropriate functions $f$ and $g$ into (3.2.10), we will derive a recursive formula for the convolution sum $S_{a, b}(n)$.

Theorem 3.3.1. Let $a$ and $b$ be odd positive integers. Then for all $n \in \mathbb{N}$, we have

$$
\begin{aligned}
2 S_{a, b}(n)=2 & \sum_{\substack{\imath=0 \\
\imath \equiv 0(\bmod 2)}}^{a}\left(\begin{array}{l}
a \\
\imath
\end{array}\right) S_{a-\imath, b+\imath}(n)+2 \sum_{\substack{\imath=0 \\
\imath \equiv 0(\bmod 2)}}^{b}\left(\begin{array}{l}
b \\
\imath
\end{array}\right) S_{b-\imath, a+\imath}(n) \\
& +n \sigma_{a+b-1}(n)-\sigma_{a+b}(n)-\sum_{d \mid n} d^{a} \sum_{m=1}^{d-1} m^{b} \\
& +\sum_{\imath=1}^{b}\left(\begin{array}{l}
b \\
\imath
\end{array}\right)(-1)^{\imath} \sum_{d \mid n} d^{b-\imath} \sum_{m=1}^{d-1} m^{a+\imath}
\end{aligned}
$$

Proof. For any odd positive integers $a$ and $b$, we let $f(n)=n^{a}$ and $g(n)=n^{b}$, for all $n \in \mathbb{Z}$. Since these are odd integers, we have

$$
-f(n)=-n^{a}=(-n)^{a}=f(-n)
$$

and so both $f$ and $g$ are odd functions. We use these functions in Theorem 3.2.6. The left hand side of equation (3.2.10) yields

$$
\begin{aligned}
2 \sum_{s x+t y=n} f(s) g(t) & =2 \sum_{m=1}^{n-1} \sum_{s x=m} f(s) \sum_{t y=n-m} g(t) \\
& =2 \sum_{m=1}^{n-1} \sum_{d \mid m} f(d) \sum_{d \mid n-m} g(d) \\
& =2 \sum_{m=1}^{n-1} \sigma_{a}(m) \sigma_{b}(n-m) \\
& =2 S_{a, b}(n) .
\end{aligned}
$$


We evaluate the right hand side of equation (3.2.10) term by term. To start, we obtain

$$
\begin{aligned}
\sum_{d \mid n}\left(\frac{n}{d}-1\right) f(d) g(d) & =\sum_{d \mid n}\left(\frac{n}{d}-1\right) d^{a+b} \\
& =n \sum_{d \mid n} d^{a+b-1}-\sum_{d \mid n} d^{a+b} \\
& =n \sigma_{a+b-1}(n)-\sigma_{a+b}(n)
\end{aligned}
$$

Next, we have

$$
\begin{aligned}
\sum_{s x+t y=n} f(t+s) g(s) & =\sum_{m=1}^{n-1} \sum_{s x=m} \sum_{t y=n-m} f(t+s) g(s) \\
& =\sum_{m=1}^{n-1} \sum_{s x=m} \sum_{t y=n-m} \sum_{\imath=0}^{a}\left(\begin{array}{l}
a \\
\imath
\end{array}\right) t^{a-\imath} s^{b+\imath} \\
& =\sum_{\imath=0}^{a}\left(\begin{array}{l}
a \\
\imath
\end{array}\right) \sum_{m=1}^{n-1} \sum_{s x=m} s^{b+\imath} \sum_{t y=n-m} t^{a-\imath} \\
& =\sum_{\imath=0}^{a}\left(\begin{array}{l}
a \\
\imath
\end{array}\right) S_{a-\imath, b+\imath}(n) .
\end{aligned}
$$

And by similar reasoning we deduce

$$
\begin{aligned}
\sum_{s x+t y=n} f(t-s) g(s) & =\sum_{\imath=0}^{a}\left(\begin{array}{l}
a \\
i
\end{array}\right)(-1)^{\imath} S_{a-\imath, b+\imath}(n), \\
\sum_{s x+t y=n} g(t+s) f(s) & =\sum_{\imath=0}^{b}\left(\begin{array}{l}
b \\
\imath
\end{array}\right) S_{b-\imath, a+\imath}(n), \\
\sum_{s x+t y=n} g(t-s) f(s) & =\sum_{\imath=0}^{b}\left(\begin{array}{l}
b \\
\imath
\end{array}\right)(-1)^{\imath} S_{b-\imath, a+\imath}(n) .
\end{aligned}
$$


When we add (3.32) and (3.3.3), we obtain

$$
\sum_{\imath=0}^{a}\left(\begin{array}{l}
a \\
\imath
\end{array}\right) S_{a-\imath, b+\imath}(n)\left(1+(-1)^{\imath}\right)=2 \sum_{\substack{\imath=0 \\
\imath \equiv 0(\bmod 2)}}^{a}\left(\begin{array}{l}
a \\
\imath
\end{array}\right) S_{a-\imath, b+\imath}(n)
$$

Similarly, adding (3.3.4) and (3.3.5) gives

$$
\sum_{n=1}^{\infty} q\left(2 \sum_{\substack{\imath=0 \\
\imath \equiv 0(\bmod 2)}}^{b}\left(\begin{array}{l}
b \\
\imath
\end{array}\right) S_{b-\imath, a+\imath}(n)\right)
$$

Next, we have

$$
\begin{aligned}
& -\sum_{d \mid n} g(d) \sum_{m=1}^{d-1} f(m)=-\sum_{d \mid n} d^{b} \sum_{m=1}^{d-1} m^{a} \\
& -\sum_{d \mid n} f(d) \sum_{m=1}^{d-1} g(m)=-\sum_{d \mid n} d^{a} \sum_{m=1}^{d-1} m^{b}
\end{aligned}
$$

Finally, we have that

$$
\sum_{d \mid n} \sum_{m=1}^{d-1} f(m) g(d-m)=\sum_{\imath=0}^{b}\left(\begin{array}{l}
b \\
\imath
\end{array}\right)(-1)^{\imath} \sum_{d \mid m} d^{b-\imath} \sum_{m=1}^{d-1} m^{a+\imath}
$$

Thus, by Theorem 3.2.6, when we add together (3.3.1), (3.3.6), (3.3.7), (3.3.8), (3.3.9) and (3.3.10), we obtain

$$
\begin{aligned}
2 S_{a, b}(n)= & 2 \sum_{\substack{\imath=0 \\
\imath \equiv 0(\bmod 2)}}^{a}\left(\begin{array}{l}
a \\
\imath
\end{array}\right) S_{a-\imath, b+\imath}(n)+2 \sum_{\substack{\imath=0 \\
\imath \equiv 0(\bmod 2)}}^{b}\left(\begin{array}{l}
b \\
\imath
\end{array}\right) S_{b-\imath, a+\imath}(n)+n \sigma_{a+b-1}(n) \\
& -\sigma_{a+b}(n)-\sum_{d \mid n} d^{b} \sum_{m=1}^{d-1} m^{a}-\sum_{d \mid n} d^{a} \sum_{m=1}^{d-1} m^{b} \\
& +\sum_{\imath=0}^{b}\left(\begin{array}{l}
b \\
\imath
\end{array}\right)(-1)^{\imath} \sum_{d \mid n} d^{b-\imath} \sum_{m=1}^{d-1} m^{a+\imath}
\end{aligned}
$$


We make the obvious simplification and complete the proof of the theorem.

It is clear from (2.4.1) that, for any $n \in \mathbb{N}$, we have $S_{a, b}(n)=S_{b, a}(n)$, for any odd positive integers $a$ and $b$. This means we may equate the right hand side of Theorem 3.3.1 with it's same expression, save the $a$ and $b$ are reversed, that is,

$$
\begin{aligned}
-\sum_{d \mid n} d^{a} \sum_{m=1}^{d-1} m^{b} & +\sum_{\imath=1}^{b}\left(\begin{array}{l}
b \\
\imath
\end{array}\right)(-1)^{\imath} \sum_{d \mid n} d^{b-\imath} \sum_{m=1}^{d-1} m^{a+\imath} \\
& =-\sum_{d \mid n} d^{b} \sum_{m=1}^{d-1} m^{a}+\sum_{\imath=1}^{a}\left(\begin{array}{l}
a \\
\imath
\end{array}\right)(-1)^{\imath} \sum_{d \mid n} d^{a-\imath} \sum_{m=1}^{d-1} m^{b+\imath}
\end{aligned}
$$

This leads us to conclude the following corollary.

Corollary 3.3.2. Let $a$ and $b$ be odd positive integers. Then for all $n \in \mathbb{N}$ we have

$$
\sum_{\imath=0}^{a}\left(\begin{array}{l}
a \\
i
\end{array}\right)(-1)^{\imath} \sum_{d \mid n} d^{a-\imath} \sum_{m=1}^{d-1} m^{b+\imath}=\sum_{\imath=0}^{b}\left(\begin{array}{l}
b \\
\imath
\end{array}\right)(-1)^{\imath} \sum_{d \mid n} d^{b-\imath} \sum_{m=1}^{d-1} m^{a+\imath}
$$

\subsection{Other Lambert Series Products}

Our primary mathematical tool used to prove Theorem 3.3.1 was Theorem 3.1.3, given in [2, p. 7]. Theorem 3.1.3 gave a new expression for the product of Lambert series with associated cosine functions. In fact, there is another theorem in $[2$, p. 7$]$ which gives a new expression for the product of Lambert series with associated sine functions.

Theorem 3.4.1. For even functions $f, g: \mathbb{Z} \rightarrow \mathbb{C}$ satısfynng $|f(n)| \leq A n^{r},|g(n)| \leq$ 
$B n^{s}$ for some $A>0, B>0, r \geq 0, s \geq 0$, then

$$
2\left(\sum_{n=1}^{\infty} u_{n} f(n) \sin n \theta\right)\left(\sum_{n=1}^{\infty} u_{n} g(n) \sin n \theta\right)=\sum_{n=1}^{\infty} f(n) g(n) u_{n}^{2}+\sum_{k=1}^{\infty} c_{k} \cos k \theta
$$

where, for $k \in \mathbb{N}$,

$$
\begin{aligned}
c_{k}= & u_{k} \sum_{n=1}^{\infty}[f(n+k) g(n)+f(n) g(n+k)] u_{n} \\
& -u_{k} \sum_{n=1}^{\infty}[f(n) g(n-k)+f(n-k) g(n)] u_{n} \\
& -\sum_{n=k+1}^{\infty}[f(n) g(n-k)+f(n-k) g(n)] u_{n}-u_{k} \sum_{n=1}^{k-1} f(n) g(k-n) \\
& +u_{k}^{2}[f(k) g(0)+f(0) g(k)] .
\end{aligned}
$$

The proof of this theorem is given in [2, pp. 25-27] and is similar to the proof of Theorem 3.1.3. With Theorem 3.4.1, we cannot choose $\theta$ so that all the sine terms reduce to 1 . If we fix $\theta=\frac{\pi}{2}$, then the sine terms will be either 1 or -1 depending on whether $n \equiv 1(\bmod 4)$ or $n \equiv 3(\bmod 4)$, respectively. Additionally, the cosine term will be 1 or -1 corresponding to $n \equiv 0(\bmod 4)$ or $n \equiv 2(\bmod 4)$, respectively. Thus, despite simlarities to our earlier theorem, difficulties are introduced as we must take all these trigonometrıc terms into account.

We have the following relevant lemma.

Lemma 3.4.2. For any function $f: \mathbb{Z} \rightarrow \mathbb{C}$ and $\theta=\frac{\pi}{2}$ we have

$$
\sum_{n=1}^{\infty} u_{n} f(n) \sin n \theta=\sum_{n=1}^{\infty} q^{n} \sum_{\substack{d \mid n \\ d \equiv 1(\bmod 2)}}(-1)^{\frac{d-1}{2}} f(d)
$$


Proof. From Lemma 3.1.1, we have

$$
\sum_{n=1}^{\infty} u_{n} f(n) \sin n \theta=\sum_{n=1}^{\infty} q^{n} \sum_{d \mid n} f(d) \sin d \theta
$$

If $d$ is even, then $\sin d \theta=0$, so we can restrict the inner sum to odd divisors $d$ of $n$. From our earlier discussion, if $d \equiv 1$ or $3(\bmod 4)$ then $\sin d \theta$ will be 1 and -1 , respectively, so that the sine term may be expressed as $(-1)^{(d-1) / 2}$. Putting this together, we obtain the assertion of the lemma.

As we are not interested in other trigonometric cases, we fix $\theta=\frac{\pi}{2}$ for the remainder of this section. With $\theta$ now fixed, we can define the following modified divisor functions.

Definition 3.4.3. For any positive integer $a$, and any $n \in \mathbb{N}$, we let

$$
\begin{aligned}
& \underline{d}^{a}(n)=\sum_{\substack{d \mid n \\
d \equiv 1(\bmod 2)}}(-1)^{\frac{d-1}{2}} d^{a}, \\
& \bar{d}^{a}(n)=\sum_{\substack{d \mid n \\
d \equiv 0(\bmod 2)}}(-1)^{\frac{d}{2}} d^{a} .
\end{aligned}
$$

We note that these modified divisor functions are similar, but not equivalent, to those studied by Glassher [10], [11], [12] and Hahn [15].

Definition 3.4.4. For any positive integers $a$ and $b$, and $n \in \mathbb{N}$, we have

$$
\begin{aligned}
& D_{a, b}(n)=\sum_{m=1}^{n-1} \underline{d}^{a}(m) \underline{d}^{b}(n-m), \\
& F_{a, b}(n)=\sum_{m=1}^{n-1} \bar{d}^{a}(m) \sigma_{b}(n-m) .
\end{aligned}
$$

Suppose now we let $f(n)=n^{a}$ and $g(n)=n^{b}$ in Theorem 3.4.1, where $a$ and $b$ are 
any even positive integers. Then we can give a recursive formula for $D_{a, b}(n)$ similar to the formula given in Theorem 3.3.1 for $S_{a, b}(n)$.

With our functions $f$ and $g$ specified, we may write the left hand side of (3.4.1) as

$$
\begin{aligned}
2\left(\sum_{n=1}^{\infty} u_{n} n^{a} \sin n \theta\right)\left(\sum_{n=1}^{\infty} u_{n} n^{b} \sin n \theta\right) & =2 \sum_{n=1}^{\infty} q^{n} \underline{d}^{a}(n) \sum_{n=1}^{\infty} q^{n} \underline{d}^{b}(n) \\
& =\sum_{n=1}^{\infty} q^{n} \cdot 2 D_{a, b}(n)
\end{aligned}
$$

We can see that the terms of the right hand side of $(34.1)$ (which encompasses those of (3.4.2)) will be very similar to those terms of Theorem 3.3.1. The chief difference here is we must account for the cosine terms at all times.

Theorem 3.4.5. Let $a$ and $b$ be any even positive integers. Then for all $n \in \mathbb{N}$, we have

$$
\begin{aligned}
2 D_{a, b}(n)= & 2 \sum_{\substack{l=0 \\
\imath \equiv 1(\bmod 2)}}^{a}\left(\begin{array}{l}
a \\
\imath
\end{array}\right) F_{a-\imath, b+\imath}(n)+2 \sum_{\substack{\imath=0 \\
\imath \equiv 1(\bmod 2)}}^{b}\left(\begin{array}{l}
b \\
\imath
\end{array}\right) F_{b-\imath, a+\imath}(n) \\
& +n \sigma_{a+b-1}(n)-\sigma_{a+b}(n)-\sum_{d \mid n} d^{a} \sum_{\substack{m=1 \\
m \equiv d(\bmod 2)}}^{d-1}(-1)^{\frac{d-m}{2}} m^{b} \\
& -\sum_{d \mid n} d^{b} \sum_{\substack{m=1 \\
m \equiv d(\bmod 2)}}^{d-1}(-1)^{\frac{d-m}{2}} m^{a} \\
& -\sum_{\imath=0}^{b}\left(\begin{array}{l}
b \\
\imath
\end{array}\right)(-1)^{\imath} \sum_{\substack{d \mid n \\
d \equiv 0(\bmod 2)}}(-1)^{\frac{d}{2}} d^{b-\imath} \sum_{m=1}^{d-1} m^{a+\imath} .
\end{aligned}
$$

Proof. We evaluate the terms of the right hand side of (3.4.1), for the functions $f(n)=n^{a}, g(n)=n^{b} \quad$ As $a$ and $b$ are even positive integers, both $f$ and $g$ are even 
functions. By Lemma 3.2.1, and subsequently (3.3.1), we deduce

$$
\sum_{n=1}^{\infty} f(n) g(n) u_{n}^{2}=\sum_{n=1}^{\infty} q^{n}\left(n \sigma_{a+b-1}(n)-\sigma_{a+b}(n)\right)
$$

Using Lemma 3.2.2, we have

$$
\begin{aligned}
\sum_{n=1}^{\infty} u_{n} \cos n \theta \sum_{m=1}^{\infty} f(n+m) g(m) u_{m} & =\sum_{n=1}^{\infty} q^{n} \sum_{m=1}^{\infty} q^{m} \sum_{d^{\prime} \mid n} \cos d^{\prime} \theta \sum_{d \mid m}\left(d^{\prime}+d\right)^{a} d^{b} \\
& =\sum_{\imath=0}^{a}\left(\begin{array}{c}
a \\
\imath
\end{array}\right) \sum_{n=1}^{\infty} q^{n} \sum_{d^{\prime} \mid n} \cos d^{\prime} \theta d^{\prime a-\imath} \sum_{m=1}^{\infty} q^{m} \sigma_{b+\imath}(n) \\
& =\sum_{\imath=0}^{a}\left(\begin{array}{c}
a \\
i
\end{array}\right) \sum_{n=1}^{\infty} q^{n} \sum_{\substack{d^{\prime} \mid n \\
d \equiv 0(\bmod 2)}} d^{\prime a-\imath} \sum_{m=1}^{\infty} q^{m} \sigma_{b+\imath}(n) \\
& =\sum_{n=1}^{\infty} q^{n} \sum_{\imath=0}^{a}\left(\begin{array}{l}
a \\
\imath
\end{array}\right) F_{a-\imath, b+\imath}(n) .
\end{aligned}
$$

By the same reasoning and due to the evenness of the functions $f$ and $g$, we have

$$
\begin{aligned}
\sum_{n=1}^{\infty} u_{n} \cos n \theta \sum_{m=1}^{\infty} f(m-n) g(m) u_{m} & =\sum_{n=1}^{\infty} u_{n} \cos n \theta \sum_{m=1}^{\infty} f(n-m) g(m) u_{m} \\
& =\sum_{n=1}^{\infty} q^{n} \sum_{\imath=0}^{a}\left(\begin{array}{c}
a \\
\imath
\end{array}\right)(-1)^{\imath} F_{a-\imath, b+\imath}(n) .
\end{aligned}
$$

Thus,

$$
\begin{aligned}
\sum_{n=1}^{\infty} u_{n} \cos n \theta \sum_{m=1}^{\infty} u_{m}[f(n+m) g(m) & -f(n-m) g(m)] \\
& =\sum_{n=1}^{\infty} q^{n}\left(2 \sum_{\substack{\imath=1 \\
\imath \equiv 1(\bmod 2)}}^{a}\left(\begin{array}{l}
a \\
\imath
\end{array}\right) F_{a-\imath, b+\imath}(n)\right),
\end{aligned}
$$


and by similar reasoning

$$
\begin{aligned}
\sum_{n=1}^{\infty} u_{n} \cos n \theta \sum_{m=1}^{\infty} u_{m}[g(n+m) f(m) & -g(n-m) f(m)] \\
& =\sum_{n=1}^{\infty} q^{n}\left(2 \sum_{\substack{\imath=1 \\
\imath \equiv 1(\bmod 2)}}^{b}\left(\begin{array}{l}
b \\
\imath
\end{array}\right) F_{b-\imath, a+\imath}(n)\right)
\end{aligned}
$$

By Lemma 3.23 , we have

$$
\begin{aligned}
\sum_{n=1}^{\infty} \cos n \theta \sum_{m=n+1}^{\infty} f(m-n) g(m) u_{m} & =\sum_{n=1}^{\infty} \cos n \theta \sum_{m=n+1}^{\infty} f(n-m) g(m) \\
& =\sum_{n=1}^{\infty} q^{n} \sum_{d \mid n} g(d) \sum_{m=1}^{d-1} f(-m) \cos (d-m) \theta \\
& =\sum_{n=1}^{\infty} q^{n} \sum_{d \mid n} d^{b} \sum_{m=1}^{d-1} m^{a} \cos (d-m) \theta \\
& =\sum_{n=1}^{\infty} q^{n} \sum_{d \mid n} d^{b} \sum_{\substack{m=1 \\
m=d(\bmod 2)}}^{d-1}(-1)^{(d-m) / 2} m^{a} .
\end{aligned}
$$

Hence,

$$
\begin{aligned}
& -\sum_{n=1}^{\infty} \cos n \theta \sum_{m=n+1}^{\infty} u_{m}[f(m-n) g(m)+f(m) g(m-n)] \\
& \quad=-\sum_{n=1}^{\infty} q^{n}\left(\sum_{d \mid n} d^{a} \sum_{\substack{m=1 \\
m \equiv d(\bmod 2)}}^{d-1}(-1)^{(d-m) / 2} m^{b}+\sum_{d \mid n} d^{b} \sum_{\substack{m=1 \\
m \equiv d(\bmod 2)}}^{d-1}(-1)^{(d-m) / 2} m^{a}\right) .
\end{aligned}
$$


Finally, from Lemma 3.2.4, we conclude

$$
\begin{aligned}
-\sum_{n=1}^{\infty} u_{n} \cos n \theta & \sum_{m=1}^{n-1} f(m) g(m-n) \\
& =-\sum_{n=1}^{\infty} q^{n} \sum_{d \mid n} \cos d \theta \sum_{m=1}^{d-1} f(m) g(d-m) \\
& =-\sum_{n=1}^{\infty} q^{n} \sum_{\substack{d \mid n \\
d \equiv 0(\bmod 2)}}(-1)^{d / 2} \sum_{m=1}^{d-1} m^{a} \sum_{\imath=0}^{b}\left(\begin{array}{l}
b \\
\imath
\end{array}\right)(-1)^{\imath} d^{b-\imath} m^{2} \\
& =-\sum_{n=1}^{\infty} q^{n} \sum_{\imath=0}^{b}\left(\begin{array}{l}
b \\
\imath
\end{array}\right)(-1)^{\imath} \sum_{\substack{d \mid n \\
d \equiv 0(\bmod 2)}}(-1)^{d / 2} d^{b-\imath} \sum_{m=1}^{d-1} m^{a+\imath}
\end{aligned}
$$

Note that $u_{n}^{2}[f(n) g(0)+f(0) g(n)]=0$, so we can see that the right hand side of (3.4.1) can be expressed as the sum of terms (3.4.9), (3.4.10), (3.4.11), (3 4.12) and (3.4.13). As these are all power series, we group them together, and equate the resulting coefficients with the coefficients of the power series given in (3.4.8). This is exactly the statement of the theorem.

By (3.4.6) and (3.4.7) we see that, for all $n \in \mathbb{N}$ we have $D_{a, b}(n)=D_{b, a}(n)$ and $F_{a, b}(n)=F_{b, a}(n)$, for arbitrary even positive integers $a$ and $b$. In particular, we may use Theorem 3.4.5 and the symmetry of $D_{a, b}(n)$ to deduce the following corollary.

Corollary 3.4.6. Let $a$ and $b$ be any even positive integers. Then for all $n \in \mathbb{N}$, we have

$$
\begin{aligned}
& \sum_{\imath=0}^{a}\left(\begin{array}{l}
a \\
\imath
\end{array}\right)(-1)^{\imath} \sum_{\substack{d \mid n \\
d \equiv 0(\bmod 2)}}(-1)^{\frac{d}{2}} d^{a-\imath} \sum_{m=1}^{d-1} m^{b+\imath} \\
&=\sum_{\imath=0}^{b}\left(\begin{array}{l}
b \\
\imath
\end{array}\right)(-1)^{\imath} \sum_{\substack{d \mid n \\
d \equiv 0(\bmod 2)}}(-1)^{\frac{d}{2}} d^{b-\imath} \sum_{m=1}^{d-1} m^{a+\imath}
\end{aligned}
$$


Suppose we wish to evaluate Theorem 3.4.5 for specıfic even positive integers $a$ and $b$. Notice that the inner sums of the terms

$$
\sum_{d \mid n} d^{a} \sum_{\substack{m=1 \\ m \equiv d(\bmod 2)}}^{d-1}(-1)^{\frac{d-m}{2}} m^{b} \text { and } \sum_{d \mid n} d^{b} \sum_{\substack{m=1 \\ m \equiv d(\bmod 2)}}^{d-1}(-1)^{\frac{d-m}{2}} m^{a}
$$

are dependent on the congruence class of $d$ modulo 2 . Thus, we may write, for example,

$$
\begin{aligned}
\sum_{d \mid n} d^{a} \sum_{\substack{m=1 \\
m \equiv d(\bmod 2)}}^{d-1}(-1)^{\frac{d-m}{2}} m^{b}= & \sum_{\substack{d \mid n \\
d \equiv 0(\bmod 2)}} d^{a} \sum_{\substack{m=1 \\
m \equiv 0(\bmod 2)}}^{d-1}(-1)^{\frac{d-m}{2}} m^{b} \\
& +\sum_{\substack{d \mid n \\
d \equiv 1(\bmod 2)}} d^{a} \sum_{\substack{m=1 \\
m \equiv 1(\bmod 2)}}^{d-1}(-1)^{\frac{d-m}{2}} m^{b} \\
= & \sum_{\substack{d \mid n \\
d \equiv(\bmod 2)}}(-1)^{\frac{d}{2}} d^{a} \sum_{\imath=1}^{\frac{d}{2}-1}(-1)^{\imath}(2 \imath)^{b} \\
& +\sum_{\substack{d \mid n \\
d \equiv 1(\bmod 2)}}(-1)^{\frac{d-1}{2}} d^{a} \sum_{\imath=0}^{\frac{d-3}{2}}(-1)^{\imath}(2 \imath+1)^{b} .
\end{aligned}
$$

This necessitates the following definitions

Definition 3.4.7. For any positive integer $a$, and any $n \in \mathbb{N}$ we let

$$
\begin{aligned}
& \underline{\sigma}_{a}(n)=\sum_{\substack{d \mid n \\
d \equiv 1(\bmod 2)}} d^{a}, \\
& \bar{\sigma}_{a}(n)=\sum_{\substack{d \mid n \\
d \equiv 0(\bmod 2)}} d^{a} .
\end{aligned}
$$

With these new definitions, we evaluate Theorem 3.4 .5 for $a=b=2$. First, we 
have that

$$
\begin{aligned}
\sum_{d \mid n} d^{2} \sum_{\substack{m=1 \\
m \equiv d(\bmod 2)}}^{d-1}(-1)^{\frac{d-m}{2}} m^{2}= & \sum_{\substack{d \mid n \\
d \equiv 0(\bmod 2)}}\left(d^{3}-\frac{1}{2} d^{4}\right) \\
& +\sum_{\substack{d \mid n \\
d \equiv 1(\bmod 2)}}\left(-\frac{1}{2} d^{4}+d^{3}-\frac{1}{2}(-1)^{\frac{d-1}{2}} d^{2}\right)
\end{aligned}
$$

So by Theorem 3.4 .5 we obtain

$$
\begin{aligned}
2 D_{2,2}(n)= & 4 F_{1,3}(n)+4 F_{1,3}(n)+n \sigma_{3}(n)-\sigma_{4}(n)-2 \sum_{\substack{d \mid n \\
d \equiv 0(\bmod 2)}}\left(d^{3}-\frac{1}{2} d^{4}\right) \\
& -2 \sum_{\substack{d \mid n \\
d \equiv 1(\bmod 2)}}\left(-\frac{1}{2} d^{4}+d^{3}-\frac{1}{2}(-1)^{\frac{d-1}{2}} d^{2}\right) \\
& -\sum_{\imath=0}^{2}\left(\begin{array}{l}
2 \\
\imath
\end{array}\right)(-1)^{\imath} \sum_{\substack{d \mid n \\
d \equiv 0(\bmod 2)}}(-1)^{\frac{d}{2}} d^{2-\imath} \sum_{m=1}^{d-1} m^{2+\imath} .
\end{aligned}
$$

After simplifications, we find

$$
\begin{aligned}
2 D_{2,2}(n)= & 8 F_{1,3}(n)+n \sigma_{3}(n)-\sigma_{4}(n)-2 \bar{\sigma}_{3}(n)+\bar{\sigma}_{4}(n)+\underline{\sigma}_{4}(n)-2 \underline{\sigma}_{3}(n)+\underline{d}^{2} \\
& -\frac{1}{30} \bar{d}^{5}(n)+\frac{1}{30} \bar{d}(n) .
\end{aligned}
$$

Observe that we may write $D_{2,2}(n)$ in terms of a linear combination of divisor functions, our modified divisor functions (3.4.4), (3.4.5), (3.4.14) and (3.4.15) and the convolution sum $F_{1,3}(n)$ This implies that the divisor functions, the modified divisor functions and the convolution sums $F_{a, b}(n)$ and $D_{a, b}(n)$ are all intricately related to one another. 


\section{Chapter 4}

\section{Convolution Sum Identities}

\subsection{Linear Identities}

Ramanujan [22], [16, pp. 136-162] showed that for odd positive integers $a$ and $b$, $S_{a, b}(n)$ can be expressed as a linear combination of $\sigma_{a+b+1}(n), \sigma_{a+b-1}(n), \ldots, \sigma_{3}(n), \sigma(n)$, whenever the sum of $a$ and $b$ is equal to one of $2,4,6,8$ or 12. Following [17], we say that the convolution sum $S_{a, b}(n)$ has weight $a+b$. We see from Theorem 3.3.1 that $S_{a, b}(n)$ can be expressed in terms of other convolution sums with the same weight.

We use Theorem 3.3 .1 to evaluate $S_{a, b}(n)$ for weights $2,4,6,8$ and 12 . We may assume that $a \leq b$. We have only one solution to each of $a+b=2$ and $a+b=4$ in odd positive integers $a$ and $b$ with $a \leq b$, namely $(a, b)=(1,1)$ and $(a, b)=(1,3)$. Hence, Theorem 3.3.1 gives us

$$
\begin{aligned}
& S_{1,1}(n)=\frac{5}{12} \sigma_{3}(n)+\frac{(1-6 n)}{12} \sigma(n), \\
& S_{1,3}(n)=\frac{7}{80} \sigma_{5}(n)+\frac{(1-3 n)}{24} \sigma_{3}(n)-\frac{1}{240} \sigma(n) .
\end{aligned}
$$

There are two pairs of odd positive integers whose sum is equal to 6 , namely $(1,5)$ 
and $(3,3)$. We use Theorem 3.3.1 to obtain

$$
\begin{aligned}
& S_{1,5}(n)=-\frac{5}{3} S_{3,3}(n)+\frac{3}{56} \sigma_{7}(n)+\frac{(1-2 n)}{24} \sigma_{5}(n)-\frac{1}{72} \sigma_{3}(n)+\frac{1}{504} \sigma(n), \\
& S_{3,3}(n)=-6 S_{1,5}(n)+\frac{69}{280} \sigma_{7}(n)+\frac{(1-2 n)}{4} \sigma_{5}(n)-\frac{1}{120} \sigma_{3}(n)+\frac{1}{84} \sigma(n) .
\end{aligned}
$$

Solving these equations for $S_{1,5}(n)$ and $S_{3,3}(n)$, we deduce

$$
\begin{aligned}
& S_{1,5}(n)=\frac{5}{126} \sigma_{7}(n)+\frac{(1-2 n)}{24} \sigma_{5}(n)+\frac{1}{504} \sigma(n), \\
& S_{3,3}(n)=\frac{1}{120} \sigma_{7}(n)-\frac{1}{120} \sigma_{3}(n) .
\end{aligned}
$$

We have two pairs of odd positive integers whose sum gives 8 . These are $(1,7)$ and $(3,5)$. So by Theorem 3.3.1, we obtain

$$
\begin{aligned}
S_{1,7}(n)= & -7 S_{3,5}(n)+\frac{11}{288} \sigma_{9}(n)+\frac{(2-3 n)}{48} \sigma_{7}(n)-\frac{7}{240} \sigma_{5}(n)+\frac{1}{72} \sigma_{3}(n)-\frac{1}{480} \sigma(n), \\
S_{3,5}(n)= & -\frac{8}{11} S_{1,7}(n)+\frac{19}{1008} \sigma_{9}(n)+\frac{(2-3 n)}{66} \sigma_{7}(n)-\frac{1}{240} \sigma_{5}(n)+\frac{1}{504} \sigma_{3}(n) \\
& -\frac{1}{660} \sigma(n) .
\end{aligned}
$$

Then solving these equations for $S_{1,7}(n)$ and $S_{3,5}(n)$ yields

$$
\begin{aligned}
& S_{3,5}(n)=\frac{11}{5040} \sigma_{9}(n)-\frac{1}{240} \sigma_{5}(n)+\frac{1}{504} \sigma_{3}(n), \\
& S_{1,7}(n)=\frac{11}{480} \sigma_{9}(n)+\frac{(2-3 n)}{48} \sigma_{7}(n)-\frac{1}{480} \sigma(n) .
\end{aligned}
$$

Finally, there are three pairs of odd positive integers whose sum is 12 , namely 
$(1,11),(3,9)$ and $(5,7)$. By Theorem 3.1.1, we have the identities

$$
\begin{aligned}
S_{1,11}(n)= & \frac{5}{208} \sigma_{13}(n)+\frac{(1-n)}{24} \sigma_{11}(n)-\frac{11}{144} \sigma_{9}(n)+\frac{11}{84} \sigma_{7}(n)-\frac{11}{80} \sigma_{5}(n) \\
& +\frac{5}{72} \sigma_{3}(n)-\frac{691}{65520} \sigma(n)-\frac{55}{3} S_{3,9}(n)-66 S_{5,7}(n) \\
S_{3,9}(n)= & \frac{5}{2431} \sigma_{13}(n)+\frac{(1-n)}{170} \sigma_{11}(n)-\frac{\sigma_{9}(n)}{240}+\frac{9}{2380} \sigma_{7}(n)-\frac{27}{6800} \sigma_{5}(n) \\
& +\frac{\sigma_{3}(n)}{264}-\frac{691}{464100} \sigma(n)-\frac{12}{85} S_{1,11}(n)-\frac{162}{85} S_{5,7}(n) \\
S_{5,7}(n)= & \frac{1501}{226512} \sigma_{13}(n)+\frac{(1-n)}{44} \sigma_{11}(n)-\frac{3}{352} \sigma_{9}(n)+\frac{\sigma_{7}(n)}{504}-\frac{\sigma_{5}(n)}{480} \\
& +\frac{15}{1936} \sigma_{3}(n)-\frac{691}{120120} \sigma(n)-\frac{6}{11} S_{1,11}(n)-\frac{45}{22} S_{3,9}(n)
\end{aligned}
$$

We solve these equations for $S_{1,11}(n), S_{3,9}(n)$ and $S_{5,7}(n)$ by substituting the value of $S_{1,11}(n)$ in the 1dentity given for $S_{3,9}(n)$, and using that subsequent value in the identity given for $S_{5,7}(n)$. After solving for all three sums, we obtain

$$
\begin{aligned}
S_{1,11}(n) & =\frac{691}{65520} \sigma_{13}(n)+\frac{(1-n)}{24} \sigma_{11}(n)-\frac{691}{65520} \sigma(n), \\
S_{3,9}(n) & =\frac{1}{2640} \sigma_{13}(n)-\frac{1}{240} \sigma_{9}(n)+\frac{1}{264} \sigma_{3}(n), \\
S_{5,7}(n) & =\frac{1}{10080} \sigma_{13}(n)+\frac{1}{504} \sigma_{7}(n)-\frac{1}{480} \sigma_{5}(n) .
\end{aligned}
$$

The formulae (4.1.1), (4.1.2), (4.1.5), (4.1.6), (4.1.9), (4.1.10), (4.1.14), (4.1.15) and (4.1.16) agree with the results stated in [17], [26] and [8].

\subsection{Ramanujan's Approximation}

In 1916, Ramanujan [22], [16, pp. 136-162] studied the properties of $S_{a, b}^{*}(n)$, defined in (2.4.3), for odd positive integers $a$ and $b$ and for any integer $n \geq 2$. Specifically, he 
showed that

$$
\begin{aligned}
S_{a, b}^{*}(n)= & \frac{\Gamma(a+1) \Gamma(b+1)}{\Gamma(a+b+2)} \frac{\zeta(a+1) \zeta(b+1)}{\zeta(a+b+2)} \sigma_{a+b+1}(n) \\
& +\frac{\zeta(1-a)+\zeta(1-b)}{a+b} n \sigma_{a+b-1}(n)+E_{a, b}(n),
\end{aligned}
$$

where

$$
\begin{aligned}
& E_{a, b}(n)=0, \text { for } a+b=2,4,6,8,12 \\
& E_{a, b}(n)=O\left(n^{7}\right), \text { for } a+b=10 \\
& E_{a, b}(n)=O\left(n^{a+b-4}\right), \text { for } a+b \geq 14 .
\end{aligned}
$$

By referring to the error term of $S_{a, b}^{*}(n)$, we refer specifically to $E_{a, b}(n)$, and note that $S_{a, b}^{*}(n)=S_{a, b}(n)+\frac{1}{2} \zeta(-a) \sigma_{b}(n)+\frac{1}{2} \zeta(-b) \sigma_{a}(n)$. By using properties (2.1.2) and (2.1.3) of the Riemann zeta function, and property (2.1.1) of the Gamma function, we determine

$$
\begin{aligned}
S_{a, b}^{*}(n)= & -\frac{B_{a+1} B_{b+1}}{2 B_{a+b+2}} \frac{a+b+2}{(a+1)(b+1)} \sigma_{a+b+1}(n) \\
& +\frac{\zeta(1-a)+\zeta(1-b)}{a+b} n \sigma_{a+b-1}(n)+E_{a, b}(n) .
\end{aligned}
$$

Following Ramanujan [16, p. 150], we define

$$
E_{a, b}(1):=\frac{\zeta(-a)+\zeta(-b)}{2}-\frac{\zeta(1-a)+\zeta(1-b)}{a+b}-\frac{\Gamma(a+1) \Gamma(b+1)}{\Gamma(a+b+2)} \frac{\zeta(a+1) \zeta(b+1)}{\zeta(a+b+2)}
$$


Ramanujan [16, p. 152] also showed that

$$
E_{a, b}(n)=E_{a, b}(1) \tau(n), \quad n \geq 1
$$

when $a+b=10$, where $\tau(n)$ is the Ramanujan tau function defined in (2.1.4). We use this to solve for the convolution sums of weight 10 .

From Theorem 3.31 , we have the identities

$$
\begin{aligned}
S_{1,9}(n)= & \frac{13}{440} \sigma_{11}(n)+\left(\frac{1}{24}-\frac{n}{20}\right) \sigma_{9}(n)-\frac{\sigma_{7}(n)}{20}+\frac{\sigma_{5}(n)}{20}-\frac{\sigma_{3}(n)}{40}+\frac{\sigma(n)}{264} \\
& -12 S_{3,7}(n)-\frac{63}{5} S_{5,5}(n), \\
S_{3,7}(n)= & \frac{247}{47520} \sigma_{11}(n)+\left(\frac{5}{432}-\frac{n}{72}\right) \sigma_{9}(n)-\frac{\sigma_{7}(n)}{240}+\frac{\sigma_{5}(n)}{432}-\frac{\sigma_{3}(n)}{480}+\frac{5}{4752} \sigma(n) \\
& -\frac{5}{18} S_{1,9}(n)-\frac{7}{12} S_{5,5}(n), \\
S_{5,5}(n)= & \frac{923}{5544} \sigma_{11}(n)+\left(\frac{5}{12}-\frac{n}{2}\right) \sigma_{9}(n)-\frac{\sigma_{7}(n)}{12}+\frac{\sigma_{5}(n)}{252}-\frac{\sigma_{3}(n)}{24}+\frac{5}{132} \sigma(n) \\
& -10 S_{1,9}(n)-20 S_{3,7}(n) .
\end{aligned}
$$

From this, we deduce

$$
\begin{aligned}
& S_{1,9}(n)=\frac{13}{924} \sigma_{11}(n)+\frac{(5-6 n)}{120} \sigma_{9}(n)-\frac{1}{105} \sigma_{5}(n)+\frac{1}{264} \sigma(n)+\frac{12}{5} S_{5,5}(n), \\
& S_{3,7}(n)=\frac{13}{10080} \sigma_{11}(n)-\frac{1}{240} \sigma_{7}(n)+\frac{5}{1008} \sigma_{5}(n)-\frac{1}{480} \sigma_{3}(n)-\frac{5}{4} S_{5,5}(n) .
\end{aligned}
$$

Using (4.2.1), we have

$$
S_{5,5}(n)=\frac{65}{174132} \sigma_{11}(n)+\frac{\sigma_{5}(n)}{252}-\frac{3}{691} \tau(n) .
$$


Using this, we find

$$
\begin{aligned}
& S_{1,9}(n)=\frac{455}{30404} \sigma_{11}(n)+\frac{(5-6 n)}{120} \sigma_{9}(n)+\frac{1}{264} \sigma(n)-\frac{36}{3455} \tau(n) \\
& S_{3,7}(n)=\frac{91}{110560} \sigma_{11}(n)-\frac{1}{240} \sigma_{7}(n)-\frac{1}{480} \sigma_{3}(n)+\frac{15}{2764} \tau(n) .
\end{aligned}
$$

The identities (4.2.10), (4 2.11) and (4.2.12) agree with those given by Lahiri ([19], $(19),(20),(21))$ and by Cheng and Williams ([8], (1.12),(1.13), (1.14)). Identities (4.2.6), (4.2.7), (4.2.8) and (4.2.9) are believed to be new. Identity (4.2.5) can be deduced from Theorem 12.3 of [26]. 


\section{Chapter 5}

\section{New Convolution Sum Identities}

\subsection{New Identities}

For those convolution sums of weight $14,16,18,20$ or 24 , Ramanujan $[16, p$ 152] showed that, for odd positive integers $a$ and $b$ and for $n \in \mathbb{N}$, the error function $E_{a, b}(n)$ is given by

$$
\begin{aligned}
\sigma_{a+b-11}(0) E_{a, b}(n)= & E_{a, b}(1)\left\{\sigma_{a+b-11}(0) \tau(n)+\sigma_{a+b-11}(1) \tau(n-1)\right. \\
& \left.+\ldots+\sigma_{a+b-11}(n-1) \tau(1)\right\} \\
E_{a, b}(n)= & E_{a, b}(1) \tau(n)+\frac{2 E_{a, b}(1)}{\zeta(11-a-b)} \sum_{m=1}^{n-1} \sigma_{a+b-11}(m) \tau(n-m)
\end{aligned}
$$

Thus, by using Theorem 3.1.1 along with Ramanujan's formulae given by (4.2.2), (4.2.3) and (5.1.1), we can determine the values of those convolution sums of weight 14,16 , 18,20 and 24. We explicitly state how to solve this for convolution sums of weight 14. The identities for convolution sums of weights $16,18,20$ and 24 will be stated, with the understanding that the method used for solving those sums of weight 14 is the same for the remaining weights. 


\subsection{Weight 14}

By Theorem 3.3.1, we may write a specific convolution sum of weight 14 in terms of divisor functions and the other convolution sums of weight 14 , that is,

$$
\begin{aligned}
S_{1,13}(n)= & \frac{17}{840} \sigma_{15}(n)+\left(\frac{1}{24}-\frac{n}{28}\right) \sigma_{13}(n)-\frac{13}{120} \sigma_{11}(n)+\frac{143}{504} \sigma_{9}(n) \\
& -\frac{143}{280} \sigma_{7}(n)+\frac{13}{24} \sigma_{5}(n)-\frac{691}{2520} \sigma_{3}(n)+\frac{\sigma(n)}{24}-26 S_{3,11}(n)-143 S_{5,9}(n) \\
& -\frac{858}{7} S_{7,7}(n) \\
S_{3,11}(n)= & \frac{1819}{1812720} \sigma_{15}(n)+\left(\frac{7}{1992}-\frac{n}{332}\right) \sigma_{13}(n)-\frac{\sigma_{11}(n)}{240}+\frac{517}{83664} \sigma_{9}(n) \\
& -\frac{11}{1328} \sigma_{7}(n)+\frac{47}{3984} \sigma_{5}(n)-\frac{691}{65520} \sigma_{3}(n)+\frac{7}{1992} \sigma(n)-\frac{7}{83} S_{1,13}(n) \\
& -\frac{517}{166} S_{5,9}(n)-\frac{165}{83} S_{7,7}(n), \\
S_{5,9}(n)= & \frac{2669}{2542540} \sigma_{15}(n)+\left(\frac{7}{1524}-\frac{n}{254}\right) \sigma_{13}(n)-\frac{47}{15240} \sigma_{11}(n)+\frac{\sigma_{9}(n)}{504} \\
& -\frac{3}{2540} \sigma_{7}(n)+\frac{\sigma_{5}(n)}{264}-\frac{32477}{4160520} \sigma_{3}(n)+\frac{7}{1524} \sigma(n)-\frac{14}{127} S_{1,13}(n) \\
& -\frac{94}{127} S_{3,11}(n)-\frac{36}{127} S_{7,7}(n), \\
& \frac{12869}{102960} \sigma_{15}(n)+\left(\frac{7}{12}-\frac{n}{2}\right) \sigma_{13}(n)-\frac{7}{24} \sigma_{11}(n)+\frac{\sigma_{9}(n)}{12}-\frac{\sigma_{7}(n)}{240} \\
& \frac{7}{44} \sigma_{5}(n)-\frac{691}{936} \sigma_{3}(n)+\frac{7}{12} \sigma(n)-14 S_{1,13}(n)-70 S_{3,11}(n)-42 S_{5,9}(n) \\
S_{7,7}(n) & (5.2 .3) \\
= &
\end{aligned}
$$

Our next step is to write each of $S_{3,11}(n), S_{5,9}(n)$ and $S_{7,7}(n)$ in terms of divisor functions and $S_{1,13}(n)$. We substitute (5.2.2) into (5.2.3), then substitute the resulting value of (5.2.3) into (5 2.4). This gives us an expression for $S_{7,7}(n)$ solely in terms of divisor functions and $S_{1,13}(n)$. We then substitute this expression into (5.2.3), so that $S_{5,9}(n)$ may be written as a linear combination of divisor functions and $S_{1,13}(n)$. 
Finally, we use these two expressions in (5.2.2), giving us

$$
\begin{aligned}
S_{3,11}(n)= & \frac{17}{7560} \sigma_{15}(n)+\left(\frac{245-210 n}{22464}\right) \sigma_{13}(n)-\frac{\sigma_{11}(n)}{240}-\frac{691}{65520} \sigma_{3}(n) \\
& +\frac{245}{22464} \sigma(n)-\frac{245}{936} S_{1,13}(n) \\
S_{5,9}(n)= & -\frac{85}{108108} \sigma_{15}(n)+\left(\frac{42 n-49}{11232}\right) \sigma_{13}(n)+\frac{\sigma_{9}(n)}{504}+\frac{\sigma_{5}(n)}{264} \\
& -\frac{49}{11232} \sigma(n)+\frac{49}{468} S_{1,13}(n), \\
S_{7,7}(n)= & \frac{17}{28080} \sigma_{15}(n)+\left(\frac{35-30 n}{11232}\right) \sigma_{13}(n)-\frac{\sigma_{7}(n)}{240}+\frac{35}{11232} \sigma(n)-\frac{35}{468} S_{1,13}(n) .
\end{aligned}
$$

Next, we use Ramanujan's formula for $S_{a, b}(n)$ to evaluate the sum $S_{1,13}(n)$ By $(4.2 .2)$, we have

$$
S_{1,13}^{*}(n)=\frac{85}{10851} \sigma_{15}(n)-\frac{n \sigma_{13}(n)}{28}+E_{1,13}(n)
$$

From (4.2.3), we determine that $E_{1,13}(1)=-\frac{1404}{25319}$, and hence

$$
E_{1,13}(n)=-\frac{1404}{25319} \tau(n)-\frac{336960}{25319} \sum_{m=1}^{n-1} \sigma_{3}(m) \tau(n-m)
$$

Finally, we have that $S_{1,13}^{*}(n)=S_{1,13}(n)-\frac{1}{2} \zeta(-1) \sigma_{13}(n)-\frac{1}{2} \zeta(-13) \sigma(n)$. Putting this all together, we deduce

$$
\begin{aligned}
S_{1,13}(n)= & \frac{85}{10851} \sigma_{15}(n)+\frac{\sigma_{13}(n)}{24}-\frac{n \sigma_{13}(n)}{28}+\frac{\sigma(n)}{24}-\frac{1404}{25319} \tau(n) \\
& -\frac{336960}{25319} \sum_{m=1}^{n-1} \sigma_{3}(m) \tau(n-m) .
\end{aligned}
$$


Now, we insert this value into (5.2.5), (5.2.6) and (5.2.7) to find

$$
\begin{aligned}
S_{3,11}(n)= & \frac{11747}{59246460} \sigma_{15}(n)-\frac{\sigma_{11}}{240}(n)-\frac{691}{65520} \sigma_{3}(n)+\frac{105}{7234} \tau(n) \\
& +\frac{12600}{3617} \sum_{m=1}^{n-1} \sigma_{3}(m) \tau(n-m), \\
S_{5,9}(n)= & \frac{85}{2506581} \sigma_{15}(n)+\frac{\sigma_{9}(n)}{504}+\frac{\sigma_{5}(n)}{264}-\frac{21}{3617} \tau(n) \\
& -\frac{5040}{3617} \sum_{m=1}^{n-1} \sigma_{3}(m) \tau(n-m), \\
S_{7,7}(n)= & \frac{17}{868080} \sigma_{15}(n)-\frac{\sigma_{7}(n)}{240}+\frac{15}{3617} \tau(n)+\frac{3600}{3617} \sum_{m=1}^{n-1} \sigma_{3}(m) \tau(n-m) .
\end{aligned}
$$

It should be noted that the identities (5.2.8), (5.2.9), (5.2.10) and (5.2.11) were deducible from Ramanujan's paper [22], [16, pp. 136-162], so it seems likely that these have been previously published. However, this author has not been able to ascertain any such publication. The identities (5.2.2) through (5.2.7) are new. Identity (5.2.1) can be deduced from Theorem 12.3 of [26].

\subsection{Weight 16}

We repeat the steps used in determining the convolution sums of weight 14 . First, we find

$$
\begin{aligned}
S_{1,15}(n)= & \frac{19}{1088} \sigma_{17}(n)+\left(\frac{1}{24}-\frac{n}{32}\right) \sigma_{15}(n)-\frac{7}{48} \sigma_{13}(n)+\frac{13}{24} \sigma_{11}(n)-\frac{143}{96} \sigma_{9}(n) \\
& +\frac{65}{24} \sigma_{7}(n)-\frac{691}{240} \sigma_{5}(n)+\frac{35}{24} \sigma_{3}(n)-\frac{3617}{16320} \sigma(n)-35 S_{3,13}(n)-273 S_{5,11}(n) \\
& -715 S_{7,9}(n), \\
S_{3,13}(n)= & \frac{437}{780640} \sigma_{17}(n)+\left(\frac{2}{861}-\frac{n}{574}\right) \sigma_{15}(n)-\frac{\sigma_{13}(n)}{240}+\frac{65}{6888} \sigma_{11}(n)
\end{aligned}
$$




$$
\begin{aligned}
& -\frac{2431}{137760} \sigma_{9}(n)+\frac{221}{6888} \sigma_{7}(n)-\frac{691}{13776} \sigma_{5}(n)+\frac{\sigma_{3}(n)}{24}-\frac{3617}{292740} \sigma(n) \\
& -\frac{16}{287} S_{1,15}(n)-\frac{195}{41} S_{5,11}(n)-\frac{2431}{287} S_{7,9}(n), \\
S_{5,11}(n)= & \frac{18563}{68761056} \sigma_{17}(n)+\left(\frac{2}{1389}-\frac{n}{926}\right) \sigma_{15}(n)-\frac{35}{22224} \sigma_{13}(n)+\frac{\sigma_{11}(n)}{504} \\
& -\frac{77}{44448} \sigma_{9}(n)+\frac{35}{11112} \sigma_{7}(n)-\frac{691}{65520} \sigma_{5}(n)+\frac{175}{11112} \sigma_{3}(n)-\frac{3617}{472260} \sigma(n) \\
& -\frac{16}{463} S_{1,15}(n)-\frac{175}{463} S_{3,13}(n)-\frac{385}{463} S_{7,9}(n), \\
S_{7,9}(n)= & \frac{43757}{14391520} \sigma_{17}(n)+\left(\frac{2}{111}-\frac{n}{74}\right) \sigma_{15}(n)-\frac{119}{8880} \sigma_{13}(n)+\frac{7}{888} \sigma_{11}(n) \\
& -\frac{\sigma_{9}(n)}{480}+\frac{\sigma_{7}(n)}{264}-\frac{4837}{115440} \sigma_{5}(n)+\frac{119}{888} \sigma_{3}(n)-\frac{3617}{37740} \sigma(n)-\frac{16}{37} S_{1,15}(n) \\
& -\frac{119}{37} S_{3,13}(n)-\frac{147}{37} S_{5,11}(n) .
\end{aligned}
$$

Next, writing the sums in terms of $S_{1,15}(n)$ and divisor functions, we obtain

$$
\begin{aligned}
S_{3,13}(n)= & \frac{133}{108800} \sigma_{17}(n)+\left(\frac{44-33 n}{5760}\right) \sigma_{15}(n)-\frac{\sigma_{13}(n)}{240}+\frac{\sigma_{3}(n)}{24}-\frac{39787}{979200} \sigma(n) \\
& -\frac{11}{60} S_{1,15}(n) \\
S_{5,11}(n)= & -\frac{2831}{11880960} \sigma_{17}(n)+\left(\frac{3 n-4}{2304}\right) \sigma_{15}(n)+\frac{\sigma_{11}(n)}{504}-\frac{691}{65520} \sigma_{5}(n) \\
& +\frac{3617}{391680} \sigma(n)+\frac{1}{24} S_{1,15}(n), \\
S_{7,9}(n)= & \frac{133}{2393600} \sigma_{17}(n)+\left(\frac{4-3 n}{11520}\right) \sigma_{15}(n)-\frac{\sigma_{9}(n)}{480}+\frac{\sigma_{7}(n)}{264}-\frac{3617}{1958400} \sigma(n) \\
& -\frac{1}{120} S_{1,15}(n) .
\end{aligned}
$$

Next, we have

$$
S_{1,15}^{*}(n)=\frac{1443183}{238636480} \sigma_{17}(n)-\frac{n \sigma_{15}(n)}{32}+E_{1,15}(n)
$$


and $E_{1,15}(1)=\frac{9000}{43867}$ gives us

$$
E_{1,15}(n)=\frac{9000}{43867} \tau(n)-\frac{4536000}{43867} \sum_{m=1}^{n-1} \sigma_{5}(m) \tau(n-m) .
$$

As $S_{1,15}^{*}(n)=S_{1,15}(n)-\frac{1}{24} \sigma_{15}(n)+\frac{3617}{16320} \sigma(n)$, we deduce

$$
\begin{aligned}
S_{1,15}(n)= & \frac{1443183}{238636480} \sigma_{17}(n)+\frac{1}{24} \sigma_{15}(n)-\frac{n \sigma_{15}(n)}{32}-\frac{3617}{16320} \sigma(n) \\
& +\frac{9000}{43867} \tau(n)-\frac{4536000}{43867} \sum_{m=1}^{n-1} \sigma_{5}(m) \tau(n-m) .
\end{aligned}
$$

Then, to conclude, we substitute in our value for $S_{1,15}(n)$ into $(5.3 .5)-(5.3 .7)$ to find

$$
\begin{aligned}
S_{3,13}(n)= & \frac{399}{3509360} \sigma_{17}(n)-\frac{\sigma_{13}(n)}{240}+\frac{\sigma_{3}(n)}{24}-\frac{1650}{43867} \tau(n) \\
& +\frac{831600}{43867} \sum_{m=1}^{n-1} \sigma_{5}(m) \tau(n-m), \\
S_{5,11}(n)= & \frac{13129}{958055280} \sigma_{17}(n)+\frac{\sigma_{11}(n)}{504}-\frac{691}{65520} \sigma_{5}(n)+\frac{375}{43867} \tau(n) \\
& -\frac{189000}{43867} \sum_{m=1}^{n-1} \sigma_{5}(m) \tau(n-m), \\
S_{7,9}(n)= & \frac{399}{77205920} \sigma_{17}(n)-\frac{\sigma_{9}(n)}{480}+\frac{\sigma_{7}(n)}{264}-\frac{75}{43867} \tau(n) \\
& +\frac{37800}{43867} \sum_{m=1}^{n-1} \sigma_{5}(m) \tau(n-m) .
\end{aligned}
$$

Identities (5.3.2) - (5 3.7) are new. Identities (5.3.8) - (5.3.11) do not appear to have been previously published, but were deducible from [22], [16, pp. 136-162]. Identity (5.3.1) can be deduced from Theorem 12.3 of [26]. 


\section{$5.4 \quad$ Weight 18}

As before, we repeat similar steps to first obtain

$$
\begin{aligned}
S_{1,17}(n)= & \frac{7}{456} \sigma_{19}(n)+\left(\frac{1}{24}-\frac{n}{36}\right) \sigma_{17}(n)-\frac{17}{90} \sigma_{15}(n)+\frac{17}{18} \sigma_{13}(n)-\frac{221}{60} \sigma_{11}(n) \\
& +\frac{1105}{108} \sigma_{9}(n)-\frac{11747}{630} \sigma_{7}(n)+\frac{119}{6} \sigma_{5}(n)-\frac{3617}{360} \sigma_{3}(n)+\frac{43867}{28728} \sigma(n) \\
& -\frac{136}{3} S_{3,15}(n)-476 S_{5,13}(n)-1768 S_{7,11}(n)-\frac{12155}{9} S_{9,9}(n) \\
S_{3,15}(n)= & \frac{1211}{3534912} \sigma_{19}(n)+\left(\frac{1}{608}-\frac{n}{912}\right) \sigma_{17}(n)-\frac{\sigma_{15}(n)}{240}+\frac{37}{2736} \sigma_{13}(n) \\
& -\frac{65}{1824} \sigma_{11}(n)+\frac{455}{5472} \sigma_{9}(n)-\frac{3455}{19152} \sigma_{7}(n)+\frac{259}{912} \sigma_{5}(n)-\frac{3617}{16320} \sigma_{3}(n) \\
& +\frac{43867}{727776} \sigma(n)-\frac{3}{76} S_{1,17}(n)-\frac{259}{38} S_{5,13}(n)-\frac{325}{19} S_{7,11}(n)-\frac{5005}{456} S_{9,9}(n),
\end{aligned}
$$

$$
\begin{aligned}
S_{5,13}(n)= & \frac{791}{8558208} \sigma_{19}(n)+\left(\frac{3}{5152}-\frac{n}{2576}\right) \sigma_{17}(n)-\frac{37}{38640} \sigma_{15}(n)+\frac{\sigma_{13}(n)}{504} \\
& -\frac{13}{4480} \sigma_{11}(n)+\frac{65}{15456} \sigma_{9}(n)-\frac{691}{47040} \sigma_{7}(n)+\frac{\sigma_{5}(n)}{24}-\frac{133829}{2627520} \sigma_{3}(n) \\
& +\frac{43867}{2055648} \sigma(n)-\frac{9}{644} S_{1,17}(n)-\frac{37}{161} S_{3,15}(n)-\frac{39}{28} S_{7,11}(n)-\frac{715}{1288} S_{9,9}(n),
\end{aligned}
$$

$$
\begin{aligned}
S_{7,11}(n)= & \frac{125969}{400282272} \sigma_{19}(n)+\left(\frac{3}{1324}-\frac{n}{662}\right) \sigma_{17}(n)-\frac{5}{1986} \sigma_{15}(n)+\frac{23}{7944} \sigma_{13}(n) \\
& -\frac{\sigma_{11}(n)}{480}+\frac{5}{3972} \sigma_{9}(n)-\frac{691}{65520} \sigma_{7}(n)+\frac{161}{2648} \sigma_{5}(n)-\frac{18085}{135048} \sigma_{3}(n) \\
& +\frac{43867}{528276} \sigma(n)-\frac{18}{331} S_{1,17}(n)-\frac{200}{331} S_{3,15}(n)-\frac{483}{331} S_{5,13}(n)-\frac{55}{331} S_{9,9}(n),
\end{aligned}
$$

$$
\begin{aligned}
S_{9,9}(n)= & \frac{36951}{369512} \sigma_{19}(n)+\left(\frac{3}{4}-\frac{n}{2}\right) \sigma_{17}(n)-\frac{7}{10} \sigma_{15}(n)+\frac{\sigma_{13}(n)}{2}-\frac{3}{20} \sigma_{11}(n) \\
& +\frac{\sigma_{9}(n)}{132}-\frac{691}{910} \sigma_{7}(n)+\frac{21}{2} \sigma_{5}(n)-\frac{25319}{680} \sigma_{3}(n)+\frac{43867}{1596} \sigma(n)-18 S_{1,17}(n) \\
& -168 S_{3,15}(n)-252 S_{5,13}(n)-72 S_{7,11}(n)
\end{aligned}
$$


from which we deduce

$$
\begin{aligned}
S_{3,15}(n)= & \frac{54307}{70326144} \sigma_{19}(n)+\left(\frac{2145-1430 n}{352512}\right) \sigma_{17}(n)-\frac{\sigma_{15}}{240}-\frac{3617}{16320} \sigma_{3}(n) \\
& +\frac{31364905}{140652288} \sigma(n)-\frac{715}{4896} S_{1,17}(n) \\
S_{5,13}(n)= & -\frac{7975}{61535376} \sigma_{19}(n)+\left(\frac{242 n-363}{308448}\right) \sigma_{17}(n)+\frac{\sigma_{13}(n)}{504}+\frac{\sigma_{5}(n)}{24} \\
& -\frac{5307907}{123070752} \sigma(n)+\frac{121}{4284} S_{1,17}(n) \\
S_{7,11}(n)= & \frac{9361}{228559968} \sigma_{19}(n)+\left(\frac{15-10 n}{44064}\right) \sigma_{17}(n)-\frac{\sigma_{11}(n)}{480}-\frac{691}{65520} \sigma_{7}(n) \\
& +\frac{219335}{17581536} \sigma(n)-\frac{5}{612} S_{1,17}(n) \\
S_{9,9}(n)= & -\frac{725}{32232816} \sigma_{19}(n)+\left(\frac{2 n-3}{14688}\right) \sigma_{17}(n)+\frac{\sigma_{9}(n)}{132}-\frac{43867}{5860512} \sigma(n) \\
& +\frac{1}{204} S_{1,17}(n) .
\end{aligned}
$$

Then, we have

$$
S_{1,17}^{*}(n)=\frac{12063425}{2508112404} \sigma_{19}(n)-\frac{n}{36} \sigma_{17}(n)+E_{1,17}(n),
$$

and $E_{1,17}(1)=-\frac{269892}{174611}$ means that

$$
E_{1,17}(n)=-\frac{269892}{174611} \tau(n)-\frac{129548160}{174611} \sum_{m=1}^{n-1} \sigma_{7}(m) \tau(n-m)
$$

As we have $S_{1,17}^{*}(n)=S_{1,17}(n)+\frac{1}{2} \zeta(-1) \sigma_{17}(n)+\frac{1}{2} \zeta(-17) \sigma(n)$, we get

$$
\begin{aligned}
S_{1,17}(n)= & \frac{12063425}{2508112404} \sigma_{19}(n)+\left(\frac{1}{24}-\frac{n}{36}\right) \sigma_{17}(n)+\frac{43867}{28728} \sigma(n) \\
& -\frac{269892}{174611} \tau(n)-\frac{129548160}{174611} \sum_{m=1}^{n-1} \sigma_{7}(m) \tau(n-m) .
\end{aligned}
$$


Finally, we find

$$
\begin{aligned}
S_{3,15}(n)= & \frac{39787}{569930304} \sigma_{19}(n)-\frac{\sigma_{15}(n)}{240}-\frac{3617}{16320} \sigma_{3}(n)+\frac{315315}{1396888} \tau(n) \\
& +\frac{18918900}{174611} \sum_{m=1}^{n-1} \sigma_{7}(m) \tau(n-m) \\
S_{5,13}(n)= & \frac{275}{44001972} \sigma_{19}(n)+\frac{\sigma_{13}(n)}{504}+\frac{\sigma_{5}(n)}{24}-\frac{7623}{174611} \tau(n) \\
& -\frac{3659040}{174611} \sum_{m=1}^{n-1} \sigma_{7}(m) \tau(n-m) \\
S_{7,11}(n)= & \frac{7601}{4576205088} \sigma_{19}(n)-\frac{\sigma_{11}(n)}{480}-\frac{691}{65520} \sigma_{7}(n)+\frac{2205}{174611} \tau(n) \\
& +\frac{1058400}{174611} \sum_{m=1}^{n-1} \sigma_{7}(m) \tau(n-m) \\
S_{9,9}(n)= & \frac{25}{23048652} \sigma_{19}(n)+\frac{\sigma_{9}(n)}{132}-\frac{1323}{174611} \tau(n)-\frac{635040}{174611} \sum_{m=1}^{n-1} \sigma_{7}(m) \tau(n-m)
\end{aligned}
$$

Identities (5.4.2) - (5.4.9) are new, and identities (5.4.10) - (5.4.14) were deducible from [22], [16, pp. 136-162]. Identity (5.4.1) can be deduced from Theorem 12.3 of [26].

\subsection{Weight 20}

As before, we begin with the identities

$$
\begin{aligned}
S_{1,19}(n)= & \frac{23}{1680} \sigma_{21}(n)+\left(\frac{1}{24}-\frac{n}{40}\right) \sigma_{19}(n)-\frac{19}{80} \sigma_{17}(n)+\frac{323}{210} \sigma_{15}(n)-\frac{323}{40} \sigma_{13}(n) \\
& +\frac{4199}{132} \sigma_{11}(n)-\frac{223193}{2520} \sigma_{9}(n)+\frac{323}{2} \sigma_{7}(n)-\frac{68723}{400} \sigma_{5}(n)+\frac{43867}{504} \sigma_{3}(n) \\
& -\frac{174611}{13200} \sigma(n)-57 S_{3,17}(n)-\frac{3876}{5} S_{5,15}(n)-3876 S_{7,13}(n) \\
& -8398 S_{9,11}(n)
\end{aligned}
$$




$$
\begin{aligned}
& S_{3,17}(n)=\frac{1219}{5434380} \sigma_{21}(n)+\left(\frac{5}{4086}-\frac{n}{1362}\right) \sigma_{19}(n)-\frac{\sigma_{17}(n)}{240}+\frac{527}{28602} \sigma_{15}(n) \\
& -\frac{1819}{27240} \sigma_{13}(n)+\frac{18343}{89892} \sigma_{11}(n)-\frac{975001}{1716120} \sigma_{9}(n)+\frac{1819}{1362} \sigma_{7}(n)-\frac{112127}{54480} \sigma_{5}(n) \\
& +\frac{43867}{28728} \sigma_{3}(n)-\frac{174611}{449460} \sigma(n)-\frac{20}{681} S_{1,19}(n)-\frac{2108}{227} S_{5,15}(n)-\frac{7276}{227} S_{7,13}(n) \\
& -\frac{36686}{681} S_{9,11}(n) \\
& S_{5,15}(n)=\frac{18653}{489027168} \sigma_{21}(n)+\left(\frac{5}{18024}-\frac{n}{6008}\right) \sigma_{19}(n)-\frac{31}{48064} \sigma_{17}(n)+\frac{\sigma_{15}(n)}{504} \\
& -\frac{109}{24032} \sigma_{13}(n)+\frac{3185}{396528} \sigma_{11}(n)-\frac{4837}{216288} \sigma_{9}(n)+\frac{545}{6008} \sigma_{7}(n)-\frac{3617}{16320} \sigma_{5}(n) \\
& +\frac{6799385}{28766304} \sigma_{3}(n)-\frac{174611}{1982640} \sigma(n)-\frac{5}{751} S_{1,19}(n)-\frac{465}{3004} S_{3,17}(n) \\
& -\frac{1635}{751} S_{7,13}(n)-\frac{3185}{1502} S_{9,11}(n), \\
& S_{7,13}(n)=\frac{319769}{5590277280} \sigma_{21}(n)+\left(\frac{5}{10302}-\frac{n}{3434}\right) \sigma_{19}(n)-\frac{107}{137360} \sigma_{17}(n) \\
& +\frac{109}{72114} \sigma_{15}(n)-\frac{\sigma_{13}(n)}{480}+\frac{793}{453288} \sigma_{11}(n)-\frac{42151}{8653680} \sigma_{9}(n)+\frac{\sigma_{7}(n)}{24} \\
& -\frac{394253}{2335120} \sigma_{5}(n)+\frac{4693769}{16441992} \sigma_{3}(n)-\frac{174611}{1133220} \sigma(n)-\frac{20}{1717} S_{1,19}(n) \\
& -\frac{321}{1717} S_{3,17}(n)-\frac{1308}{1717} S_{5,15}(n)-\frac{793}{1717} S_{9,11}(n), \\
& S_{9,11}(n)=\frac{129329}{79008384} \sigma_{21}(n)+\left(\frac{5}{336}-\frac{n}{112}\right) \sigma_{19}(n)-\frac{83}{4480} \sigma_{17}(n)+\frac{\sigma_{15}(n)}{48} \\
& -\frac{61}{4480} \sigma_{13}(n)+\frac{\sigma_{11}(n)}{264}-\frac{691}{65520} \sigma_{9}(n)+\frac{61}{224} \sigma_{7}(n)-\frac{25319}{10880} \sigma_{5}(n) \\
& +\frac{3640961}{536256} \sigma_{3}(n)-\frac{174611}{36960} \sigma(n)-\frac{5}{14} S_{1,19}(n)-\frac{249}{56} S_{3,17}(n)-\frac{21}{2} S_{5,15}(n) \\
& -\frac{183}{28} S_{7,13}(n) \text {. }
\end{aligned}
$$

And we deduce

$$
\begin{aligned}
S_{3,17}(n)= & \frac{470971}{948024000} \sigma_{21}(n)+\left(\frac{1183}{246240}-\frac{1183 n}{410400}\right) \sigma_{19}(n)-\frac{\sigma_{17}(n)}{240} \\
& +\frac{43867}{28728} \sigma_{3}(n)-\frac{206564813}{135432000} \sigma(n)-\frac{1183}{10260} S_{1,19}(n)
\end{aligned}
$$




$$
\begin{aligned}
S_{5,15}(n)= & -\frac{532979}{8595417600} \sigma_{21}(n)+\left(\frac{91 n}{218880}-\frac{91}{131328}\right) \sigma_{19}(n)+\frac{\sigma_{15}(n)}{504} \\
& -\frac{3617}{16320} \sigma_{5}(n)+\frac{15889601}{72230400} \sigma(n)+\frac{91}{5472} S_{1,19}(n), \\
S_{7,13}(n)= & \frac{2231}{180576000} \sigma_{21}(n)+\left(\frac{41}{328320}-\frac{41 n}{547200}\right) \sigma_{19}-\frac{\sigma_{13}(n)}{480} \\
& +\frac{\sigma_{7}(n)}{24}-\frac{7159051}{180576000} \sigma(n)-\frac{41}{13680} S_{1,19}(n), \\
S_{9,11}(n)= & -\frac{28267}{16432416000} \sigma_{21}(n)+\left(\frac{7 n}{547200}-\frac{7}{328320}\right) \sigma_{19}(n)+\frac{\sigma_{11}(n)}{264} \\
& -\frac{691}{65520} \sigma_{9}(n)+\frac{1222277}{180576000} \sigma(n)+\frac{7}{13680} S_{1,19}(n) .
\end{aligned}
$$

Then we find

$$
S_{1,19}^{*}(n)=\frac{4016053}{1025415600} \sigma_{21}(n)-\frac{n \sigma_{19}(n)}{40}+E_{1,19}(n)
$$

and $E_{1,19}(1)=\frac{1026000}{77683}$ means that

$$
E_{1,19}(n)=\frac{1026000}{77683} \tau(n)-\frac{270864000}{77683} \sum_{m=1}^{n-1} \sigma_{9}(m) \tau(n-m) .
$$

With $S_{1,19}(n)=S_{1,19}^{*}(n)-\frac{1}{2} \zeta(-1) \sigma_{19}(n)-\frac{1}{2} \zeta(-19) \sigma(n)$, we deduce

$$
\begin{aligned}
S_{1,19}(n)= & \frac{4016053}{1025415600} \sigma_{21}(n)+\left(\frac{1}{24}-\frac{n}{40}\right) \sigma_{19}(n)-\frac{174611}{13200} \sigma(n) \\
& +\frac{1026000}{77683} \tau(n)-\frac{270864000}{77683} \sum_{m=1}^{n-1} \sigma_{9}(m) \tau(n-m) .
\end{aligned}
$$

Finally, we have

$$
S_{3,17}(n)=\frac{1008941}{22316772240} \sigma_{21}(n)-\frac{\sigma_{17}(n)}{240}+\frac{43867}{28728} \sigma_{3}(n)-\frac{118300}{77683} \tau(n)
$$


CHAPTER 5. NEW CONVOLUTION SUM IDENTITIES

$$
\begin{aligned}
& +\frac{31231200}{77683} \sum_{m=1}^{n-1} \sigma_{9}(m) \tau(n-m), \\
S_{5,15}(n)= & \frac{83191}{26623517760} \sigma_{21}(n)+\frac{\sigma_{15}(n)}{504}-\frac{3617}{16320} \sigma_{5}(n)+\frac{34125}{155366} \tau(n) \\
& -\frac{4504500}{77683} \sum_{m=1}^{n-1} \sigma_{9}(m) \tau(n-m), \\
S_{7,13}(n)= & \frac{23}{37287840} \sigma_{21}(n)-\frac{\sigma_{13}(n)}{480}+\frac{\sigma_{7}(n)}{24}-\frac{3075}{77683} \tau(n) \\
& +\frac{811800}{77683} \sum_{m=1}^{n-1} \sigma_{9}(m) \tau(n-m), \\
S_{9,11}= & \frac{15893}{55987691760} \sigma_{21}(n)+\frac{\sigma_{11}(n)}{264}-\frac{691}{65520} \sigma_{9}(n)+\frac{525}{77683} \tau(n) \\
& -\frac{138600}{77683} \sum_{m=1}^{n-1} \sigma_{9}(m) \tau(n-m) .
\end{aligned}
$$

Identities (5.5.2) - (5 5.9) are new, and those identities (5.5.10) - (5.5.14) were deducible from [22], [16, pp. 136-162]. Identity (5.5.1) can be deduced from Theorem 12.3 of $[26]$.

\subsection{Weight 24}

We have the identities

$$
\begin{aligned}
S_{1,23}(n)= & \frac{9}{800} \sigma_{25}(n)+\left(\frac{1}{24}-\frac{n}{48}\right) \sigma_{23}(n)-\frac{253}{720} \sigma_{21}(n)+\frac{253}{72} \sigma_{19}(n)-\frac{4807}{160} \sigma_{17}(n) \\
& +\frac{7429}{36} \sigma_{15}(n)-\frac{5133439}{4680} \sigma_{13}(n)+\frac{52003}{12} \sigma_{11}(n)-\frac{17386919}{1440} \sigma_{9}(n) \\
& +\frac{11098351}{504} \sigma_{7}(n)-\frac{28112371}{1200} \sigma_{5}(n)+\frac{854513}{72} \sigma_{3}(n)-\frac{236364091}{131040} \sigma(n) \\
& -\frac{253}{3} S_{3,21}(n)-1771 S_{5,19}(n)-14421 S_{7,17}(n)-\frac{163438}{3} S_{9,15}(n) \\
& -104006 S_{11,13}(n), \\
S_{3,21}(n)= & \frac{1359}{12245200} \sigma_{25}(n)+\left(\frac{1}{1331}-\frac{n}{2662}\right) \sigma_{23}(n)-\frac{\sigma_{21}(n)}{240}+\frac{89}{2904} \sigma_{19}(n)
\end{aligned}
$$




$$
\begin{aligned}
& -\frac{741}{3872} \sigma_{17}(n)+\frac{15827}{15972} \sigma_{15}(n)-\frac{9150913}{2076360} \sigma_{13}(n)+\frac{92701}{5324} \sigma_{11}(n) \\
& -\frac{3367427}{58080} \sigma_{9}(n)+\frac{2851355}{20328} \sigma_{7}(n)-\frac{108782653}{532400} \sigma_{5}(n)+\frac{77683}{552} \sigma_{3}(n) \\
& -\frac{236364091}{7267260} \sigma(n)-\frac{24}{1331} S_{1,23}(n)-\frac{1869}{121} S_{5,19}(n)-\frac{11115}{121} S_{7,17}(n) \\
& -\frac{31654}{121} S_{9,15}(n)-\frac{556206}{1331} S_{11,13}(n) \\
& S_{5,19}(n)=\frac{76743}{8237983600} \sigma_{25}(n)+\left(\frac{1}{11629}-\frac{n}{23258}\right) \sigma_{23}(n)-\frac{979}{2790960} \sigma_{21}(n)+\frac{\sigma_{19}(n)}{504} \\
& -\frac{16853}{1860640} \sigma_{17}(n)+\frac{48127}{1535028} \sigma_{15}(n)-\frac{11829229}{126988680} \sigma_{13}(n)+\frac{17119}{46516} \sigma_{11}(n) \\
& -\frac{10239727}{5581920} \sigma_{9}(n)+\frac{38910029}{5861016} \sigma_{7}(n)-\frac{174611}{13200} \sigma_{5}(n)+\frac{76051657}{6419208} \sigma_{3}(n) \\
& -\frac{236364091}{63494340} \sigma(n)-\frac{24}{11629} S_{1,23}(n)-\frac{979}{11629} S_{3,21}(n)-\frac{50559}{11629} S_{7,17}(n) \\
& -\frac{96254}{11629} S_{9,15}(n)-\frac{102714}{11629} S_{11,13}(n) \\
& S_{7,17}(n)=\frac{28931}{6232756200} \sigma_{25}(n)+\left(\frac{1}{19449}-\frac{n}{38898}\right) \sigma_{23}(n)-\frac{143}{933552} \sigma_{21}(n) \\
& +\frac{887}{1400328} \sigma_{19}(n)-\frac{\sigma_{17}(n)}{480}+\frac{12223}{2567268} \sigma_{15}(n)-\frac{364157}{45510660} \sigma_{13}(n) \\
& +\frac{3689}{116694} \sigma_{11}(n)-\frac{2600623}{9335520} \sigma_{9}(n)+\frac{43867}{28728} \sigma_{7}(n)-\frac{1084159699}{256726800} \sigma_{5}(n) \\
& +\frac{55543345}{10735848} \sigma_{3}(n)-\frac{236364091}{106191540} \sigma(n)-\frac{8}{6483} S_{1,23}(n)-\frac{715}{19449} S_{3,21}(n) \\
& -\frac{6209}{19449} S_{5,19}(n)-\frac{24446}{19449} S_{9,15}(n)-\frac{14756}{19449} S_{11,13}(n) \\
& S_{9,15}(n)=\frac{2655867}{163634125600} \sigma_{25}(n)+\left(\frac{1}{5006}-\frac{n}{10012}\right) \sigma_{23}(n)-\frac{539}{1201440} \sigma_{21}(n) \\
& +\frac{149}{120144} \sigma_{19}(n)-\frac{2157}{800960} \sigma_{17}(n)+\frac{\sigma_{15}(n)}{264}-\frac{4837}{1561872} \sigma_{13}(n) \\
& +\frac{245}{20024} \sigma_{11}(n)-\frac{3617}{16320} \sigma_{9}(n)+\frac{31540373}{15979152} \sigma_{7}(n)-\frac{182119273}{22026400} \sigma_{5}(n) \\
& +\frac{41871137}{2763312} \sigma_{3}(n)-\frac{236364091}{27332760} \sigma(n)-\frac{12}{2503} S_{1,23}(n)-\frac{539}{5006} S_{3,21}(n) \\
& -\frac{3129}{5006} S_{5,19}(n)-\frac{6471}{5006} S_{7,17}(n)-\frac{735}{2503} S_{11,13}(n), \\
& S_{11,13}(n)=\frac{3219233}{3286589600} \sigma_{25}(n)+\left(\frac{1}{79}-\frac{n}{158}\right) \sigma_{23}(n)-\frac{451}{18960} \sigma_{21}(n)+\frac{583}{13272} \sigma_{19}(n) \\
& -\frac{341}{6320} \sigma_{17}(n)+\frac{35}{948} \sigma_{15}(n)-\frac{691}{65520} \sigma_{13}(n)+\frac{\sigma_{11}(n)}{24}-\frac{278509}{128928} \sigma_{9}(n)
\end{aligned}
$$




$$
\begin{aligned}
& +\frac{14958647}{378252} \sigma_{7}(n)-\frac{9254383}{31600} \sigma_{5}(n)+\frac{35035033}{43608} \sigma_{3}(n)-\frac{236364091}{431340} \sigma(n) \\
& -\frac{24}{79} S_{1,23}(n)-\frac{451}{79} S_{3,21}(n)-\frac{1749}{79} S_{5,19}(n)-\frac{2046}{79} S_{7,17}(n)-\frac{770}{79} S_{9,15}(n)
\end{aligned}
$$

We deduce that

$$
\begin{aligned}
S_{3,21}(n)= & \frac{127643}{542505600} \sigma_{25}(n)+\left(\frac{323}{99360}-\frac{323 n}{198720}\right) \sigma_{23}(n)-\frac{\sigma_{21}(n)}{240} \\
& +\frac{77683}{552} \sigma_{3}(n)-\frac{76345601393}{542505600} \sigma(n)-\frac{323}{4140} S_{1,23}(n) \\
S_{5,19}(n)= & -\frac{1599523}{83545862400} \sigma_{25}(n)+\left(\frac{85 n}{556416}-\frac{85}{278208}\right) \sigma_{23}(n)+\frac{\sigma_{19}(n)}{504} \\
& -\frac{174611}{13200} \sigma_{5}(n)+\frac{4018189547}{303803136} \sigma(n)+\frac{85}{11592} S_{1,23}(n) \\
S_{7,17}(n)= & \frac{50173}{20615212800} \sigma_{25}(n)+\left(\frac{7}{198720}-\frac{7 n}{397440}\right) \sigma_{23}(n)-\frac{\sigma_{17}(n)}{480} \\
& +\frac{43867}{28728} \sigma_{7}(n)-\frac{236364091}{155001600} \sigma(n)-\frac{7}{8280} S_{1,23}(n) \\
S_{9,15}(n)= & -\frac{60967}{202897094400} \sigma_{25}(n)+\left(\frac{n}{397440}-\frac{1}{198720}\right) \sigma_{23}(n)+\frac{\sigma_{15}(n)}{264} \\
& -\frac{3617}{16320} \sigma_{9}(n)+\frac{236364091}{1085011200} \sigma(n)+\frac{1}{8280} S_{1,23}(n) \\
& +\frac{37}{584236800} \sigma_{25}(n)+\left(\frac{1}{1391040}-\frac{n}{2782080}\right) \sigma_{23}(n)-\frac{691}{65520} \sigma_{13}(n) \\
S_{11,13}(n) & \frac{\sigma_{11}(n)}{24}-\frac{236364091}{7595078400} \sigma(n)-\frac{1}{57960} S_{1,23}(n) .
\end{aligned}
$$

Then we have

$$
S_{1,23}^{*}(n)=\frac{236364091}{86215278240} \sigma_{25}(n)-\frac{n \sigma_{23}(n)}{48}+E_{1,23}(n)
$$

and hence

$$
S_{1,23}(n)=\frac{236364091}{86215278240} \sigma_{25}(n)+\left(\frac{1}{24}-\frac{n}{48}\right) \sigma_{23}(n)-\frac{236364091}{131040} \sigma(n)+E_{1,23}(n)
$$


Then, as $E_{1,23}(1)=\frac{1186731000}{657931}$, we have

$$
E_{1,23}(n)=\frac{1186731000}{657931} \tau(n)-\frac{28481544000}{657931} \sum_{m=1}^{n-1} \sigma_{13}(m) \tau(n-m) .
$$

This gives us

$$
\begin{aligned}
S_{1,23}(n)= & \frac{236364091}{86215278240} \sigma_{25}(n)+\left(\frac{1}{24}-\frac{n}{48}\right) \sigma_{23}(n)-\frac{236364091}{131040} \sigma(n) \\
& +\frac{1186731000}{657931} \tau(n)-\frac{28481544000}{657931} \sum_{m=1}^{n-1} \sigma_{13}(m) \tau(n-m),
\end{aligned}
$$

and, finally we deduce

$$
\begin{aligned}
S_{3,21}(n)= & \frac{77683}{3631779120} \sigma_{25}(n)-\frac{\sigma_{21}(n)}{240}+\frac{77683}{552} \sigma_{3}(n)-\frac{92587950}{657931} \tau(n) \\
& +\frac{2222110800}{657931} \sum_{m=1}^{n-1} \sigma_{13}(m) \tau(n-m) \\
S_{5,19}(n)= & \frac{174611}{182378473200} \sigma_{25}(n)+\frac{\sigma_{19}(n)}{504}-\frac{174611}{13200} \sigma_{5}(n)+\frac{8701875}{657931} \tau(n) \\
& -\frac{208845000}{657931} \sum_{m=1}^{n-1} \sigma_{13}(m) \tau(n-m) \\
S_{7,17}(n)= & \frac{43867}{378020835360} \sigma_{25}(n)-\frac{\sigma_{17}(n)}{480}+\frac{43867}{28728} \sigma_{7}(n)-\frac{1003275}{657931} \tau(n) \\
& +\frac{24078600}{657931} \sum_{m=1}^{n-1} \sigma_{13}(m) \tau(n-m) \\
S_{9,15}(n)= & \frac{3617}{118111773120} \sigma_{25}(n)+\frac{\sigma_{15}(n)}{264}-\frac{3617}{16320} \sigma_{9}(n)+\frac{143325}{657931} \tau(n) \\
& -\frac{3439800}{657931} \sum_{m=1}^{n-1} \sigma_{13}(m) \tau(n-m) \\
S_{11,13}(n)= & \frac{691}{43107639120} \sigma_{25}(n)-\frac{691}{65520} \sigma_{13}(n)+\frac{\sigma_{11}(n)}{24}-\frac{20475}{657931} \tau(n) \\
& +\frac{491400}{657931} \sum_{m=1}^{n-1} \sigma_{13}(m) \tau(n-m) .
\end{aligned}
$$


The identities (5.6.2) - (5.6.11) are new, and the identities (5.6.12) - (5.6.17) are deducible from [22], [16, pp. 136-162]. Identity (5.6.1) can be deduced from Theorem 12.3 of $[26]$. 


\section{Chapter 6}

\section{Further Results and Future}

\section{Research Areas}

\subsection{Another Recurrence Formula}

We have the following theorem, attributed to Liouville, given in [26, p. 127].

Theorem 6.1.1. Let $k, n \in \mathbb{N}$. Then

$$
\begin{aligned}
\sum_{s=0}^{k-1}\left(\begin{array}{c}
2 k \\
2 s+1
\end{array}\right) S_{2 k-2 s-1,2 s+1}(n)= & \frac{2 k+3}{4 k+2} \sigma_{2 k+1}(n)+\left(\frac{k}{6}-n\right) \sigma_{2 k-1}(n) \\
& +\frac{1}{2 k+1} \sum_{\jmath=2}^{k}\left(\begin{array}{c}
2 k+1 \\
2 \jmath
\end{array}\right) B_{2 \jmath} \sigma_{2 k+1-2 \jmath}(n)
\end{aligned}
$$

This recurrence relation, along with Theorem 3.3.1, allows us to deduce the following theorem.

Theorem 6.1.2. Let $b$ be any odd positive integer and let $B_{n}$ denote the $n^{\text {th }}$ Bernoull 
number. Then for all $n \in \mathbb{N}$, we have

$$
\begin{aligned}
\sigma_{b+1}(n) & +\sum_{d \mid n} d \sum_{m=1}^{d-1} m^{b}+\sum_{\imath=1}^{b}\left(\begin{array}{l}
b \\
\imath
\end{array}\right)(-1)^{2+1} \sum_{d \mid n} d^{b-\imath} \sum_{m=1}^{d-1} m^{1+\imath} \\
& =\frac{b+4}{2 b+4} \sigma_{b+2}(n)+\left(\frac{b+1}{12}\right) \sigma_{b}(n)+\frac{1}{b+2} \sum_{\jmath=2}^{\frac{b+1}{2}}\left(\begin{array}{c}
b+2 \\
2 \jmath
\end{array}\right) B_{2 \jmath} \sigma_{b+2-2 \jmath}(n) .
\end{aligned}
$$

Proof. Since $b$ is an odd positive integer, there exists some $k \in \mathbb{N}$ such that $b=2 k-1$.

We use Theorem 3.3.1 with the condition that $a=1$, and note that, if $a=1$ then $2 \sum_{\substack{\imath=0 \\ \imath \equiv 0(\bmod 2)}}^{a}\left(\begin{array}{l}a \\ i\end{array}\right) S_{a-\imath, b+\imath}(n)=2 S_{1, b}(n)$. Our claim is that

$$
\sum_{s=0}^{k-1}\left(\begin{array}{c}
2 k \\
2 s+1
\end{array}\right) S_{2 k-2 s-1,2 s+1}(n)=2 \sum_{\substack{\imath=0 \\
\imath \equiv 0(\bmod 2)}}^{b}\left(\begin{array}{l}
b \\
\imath
\end{array}\right) S_{b-\imath, 1+\imath}(n)
$$

Assuming this holds for the moment, we use Theorem 3.3.1 and (6.1.1) to deduce

$$
\begin{aligned}
& -\left(n \sigma_{b}(n)-\sigma_{b+1}(n)-\sum_{d \mid n} d \sum_{m=1}^{d-1} m^{b}+\sum_{\imath=1}^{b}\left(\begin{array}{l}
b \\
\imath
\end{array}\right)(-1)^{\imath} \sum_{d \mid n} d^{b-\imath} \sum_{m=1}^{d-1} m^{1+\imath}\right) \\
& =\frac{2 k+3}{4 k+2} \sigma_{2 k+1}(n)+\left(\frac{k}{6}-n\right) \sigma_{2 k-1}(n)+\frac{1}{2 k+1} \sum_{\jmath=2}^{k}\left(\begin{array}{c}
2 k+1 \\
2 \jmath
\end{array}\right) B_{2 \jmath} \sigma_{2 k+1-2 \jmath}(n)
\end{aligned}
$$

and the proof of the theorem will follow by substituting $\frac{b+1}{2}=k$ into the right hand side of (6.1.3) and making the appropriate simplification.

Consider now the right hand sides of (3.2.4) and (3.2.5), where we take $\theta=0$, and 
$a$ and $b$ are arbitrary odd positive integers. We rewrite these terms as

$$
\sum_{n=1}^{\infty} q^{m} \sum_{m=1}^{\infty} q^{n} \sum_{d^{\prime} \mid m} g\left(d^{\prime}\right) \sum_{d \mid n} f\left(d^{\prime}+d\right)
$$

and

$$
-\sum_{n=1}^{\infty} q^{m} \sum_{m=1}^{\infty} q^{n} \sum_{d^{\prime} \mid m} g\left(d^{\prime}\right) \sum_{d \mid n} f\left(d^{\prime}-d\right)
$$

respectively. Using the same reasoning used in the proof of Theorem 3.3.1, we deduce that (6.1.4) may be written as

$$
\sum_{n=1}^{\infty} q^{n}\left(\sum_{\imath=0}^{a}\left(\begin{array}{l}
a \\
\imath
\end{array}\right) S_{a+b-\imath, \imath}(n)\right)
$$

and (6.1.5) may be written as

$$
\sum_{n=1}^{\infty} q^{n}\left(\sum_{\imath=0}^{a}\left(\begin{array}{l}
a \\
\imath
\end{array}\right)(-1)^{\imath+1} S_{a+b-\imath, \imath}(n)\right)
$$

By taking the sum of (6.16) and (6.1.7), we deduce

$$
2 \sum_{\substack{\imath=0 \\
\imath \equiv 0(\bmod 2)}}^{a}\left(\begin{array}{l}
a \\
\imath
\end{array}\right) S_{a-\imath, b+\imath}(n)=2 \sum_{\substack{\imath=0 \\
\imath \equiv 1(\bmod 2)}}^{a}\left(\begin{array}{l}
a \\
\imath
\end{array}\right) S_{a+b-\imath, \imath}(n) .
$$

From this we may conclude

$$
\sum_{\substack{l=0 \\
\imath \equiv 0(\bmod 2)}}^{a}\left(\begin{array}{l}
a \\
i
\end{array}\right) S_{a-\imath, b+\imath}(n)=\sum_{\substack{\imath=0 \\
\imath \equiv 1(\bmod 2)}}^{a}\left(\begin{array}{l}
a \\
\imath
\end{array}\right) S_{a+b-\imath, \imath}(n),
$$


and similarly,

$$
\sum_{\substack{\imath=0 \\
\imath \equiv 0(\bmod 2)}}^{b}\left(\begin{array}{l}
b \\
\imath
\end{array}\right) S_{b-\imath, a+\imath}(n)=\sum_{\substack{\imath=0 \\
\imath \equiv=(\bmod 2)}}^{b}\left(\begin{array}{l}
b \\
\imath
\end{array}\right) S_{a+b-\imath, \imath}(n)
$$

This means we may write

$$
2 \sum_{\substack{\imath=0 \\
\imath \equiv 0(\bmod 2)}}^{b}\left(\begin{array}{l}
b \\
\imath
\end{array}\right) S_{b-\imath, a+\imath}(n)=\sum_{\substack{\imath=0 \\
\imath \equiv 0(\bmod 2)}}^{b}\left(\begin{array}{l}
b \\
\imath
\end{array}\right) S_{b-\imath, a+\imath}(n)+\sum_{\substack{\imath=0 \\
\imath \equiv 1(\bmod 2)}}^{b}\left(\begin{array}{l}
b \\
\imath
\end{array}\right) S_{a+b-\imath, \imath}(n)
$$

We once again fix $a=1$ and choose $k \in \mathbb{N}$ such that $b=2 k-1$. We write the right hand side of (6.1.10) in terms of $s$ and $k$. For the first term of the right hand side of (6.1.10), we let $\imath=2 s$, so that $s$ will run from 0 to $k-1$. For the second term of the right hand side of $(6.110)$, we let $\imath=2 s+1$, and $s$ will once again run from 0 to $k-1$. Thus, the right hand side of (6.1.10) may be written as

$$
\sum_{s=0}^{k-1}\left(\begin{array}{c}
2 k-1 \\
2 s
\end{array}\right) S_{2 k-2 s-1,2 s+1}(n)+\sum_{s=0}^{k-1}\left(\begin{array}{c}
2 k-1 \\
2 s
\end{array}\right) S_{2 k-2 s-1,2 s+1}(n)
$$

By using the well known recurrence relation $\left(\begin{array}{c}n \\ m\end{array}\right)+\left(\begin{array}{c}n \\ m+1\end{array}\right)=\left(\begin{array}{c}n+1 \\ m+1\end{array}\right)$, we rewrite (6.1.11) as

$$
\sum_{s=0}^{k-1}\left(\begin{array}{c}
2 k \\
2 s+1
\end{array}\right) S_{2 k-2 s-1,2 s+1}(n)
$$

Thus, we have shown that (6.1.2) holds and we may conclude the proof of the theorem.

If we evaluate the equation given in Theorem 6.1.2 for any specific odd positive integer $b$, we see that both sides of the equation cancel with each other exactly. In 
this sense, Theorem 6.1.2 is an example of the kind of identity that may result by comparing known recursive formulae with Theorem 3.3.1.

\subsection{Bernoulli's Identity}

For $k, n \in \mathbb{N}$, Bernoulli's identity states that

$$
\sum_{m=1}^{n-1} m^{k}=\frac{1}{k+1} \sum_{\imath=0}^{k}\left(\begin{array}{c}
k+1 \\
i
\end{array}\right) B_{\imath} n^{k+1-\imath}
$$

where $B_{\imath}$ indicates the $\imath^{\text {th }}$ Bernoulli number, see for example [26, p. 48]. In light of this, we restate Theorem 3.3.1, Corollary 3.3.2, Theorem 3.4.5, Corollary 3.4.6 and Theorem 6.1 .2 , respectively.

Theorem 6.2.1. Let $a$ and $b$ be odd positive integers. Then for all $n \in \mathbb{N}$, we have

$$
\begin{aligned}
2 S_{a, b}(n)= & 2 \sum_{\substack{\imath=0 \\
\imath \equiv 0(\bmod 2)}}^{a}\left(\begin{array}{l}
a \\
\imath
\end{array}\right) S_{a-\imath, b+\imath}(n)+2 \sum_{\substack{\imath=0 \\
\imath \equiv 0(\bmod 2)}}^{b}\left(\begin{array}{l}
b \\
\imath
\end{array}\right) S_{b-\imath, a+\imath}(n)+n \sigma_{a+b-1}(n) \\
& -\sigma_{a+b}(n)-\frac{1}{b+1} \sum_{\imath=0}^{b}\left(\begin{array}{c}
b+1 \\
\imath
\end{array}\right) B_{\imath} \sigma_{a+b+1-\imath}(n) \\
& +\sum_{\imath=1}^{b}\left(\begin{array}{l}
b \\
\imath
\end{array}\right) \frac{(-1)^{\imath}}{a+i+1} \sum_{\jmath=0}^{a+\imath}\left(\begin{array}{c}
a+\imath+1 \\
\jmath
\end{array}\right) B_{\jmath} \sigma_{a+b+1-\jmath}(n) .
\end{aligned}
$$

Corollary 6.2.2. Let $a$ and $b$ be odd positive integers. Then for all $n \in \mathbb{N}$, we have

$$
\begin{aligned}
\sum_{\imath=0}^{a}\left(\begin{array}{l}
a \\
i
\end{array}\right) \frac{(-1)^{\imath}}{b+\imath+1} \sum_{\jmath=0}^{b+\imath}\left(\begin{array}{c}
b+\imath+1 \\
\jmath
\end{array}\right) B_{\jmath} \sigma_{a+b+1-\jmath}(n) \\
=\sum_{\imath=0}^{b}\left(\begin{array}{l}
b \\
\imath
\end{array}\right) \frac{(-1)^{\imath}}{a+\imath+1} \sum_{\jmath=0}^{a+\imath}\left(\begin{array}{c}
a+\imath+1 \\
j
\end{array}\right) B_{\jmath} \sigma_{a+b+1-\jmath}(n) .
\end{aligned}
$$


Theorem 6.2.3. Let $a$ and $b$ be even positive integers. Then for all $n \in \mathbb{N}$, we have

$$
\begin{aligned}
2 D_{a, b}(n)= & 2 \sum_{\substack{\imath=0 \\
\imath \equiv 1(\bmod 2)}}^{a}\left(\begin{array}{l}
a \\
\imath
\end{array}\right) F_{a-\imath, b+\imath}(n)+2 \sum_{\substack{i=0 \\
\imath \equiv 1(\bmod 2)}}^{b}\left(\begin{array}{l}
b \\
i
\end{array}\right) F_{b-\imath, a+\imath}(n)+n \sigma_{a+b-1}(n) \\
& -\sigma_{a+b}(n)-\sum_{d \mid n} d^{a} \sum_{\substack{m=1 \\
m \equiv d(\bmod 2)}}^{d-1}(-1)^{\frac{d-m}{2}} m^{b}-\sum_{d \mid n} d^{b} \sum_{\substack{m=1 \\
m \equiv d(\bmod 2)}}^{d-1}(-1)^{\frac{d-m}{2}} m^{a} \\
& -\sum_{\imath=0}^{b}\left(\begin{array}{l}
b \\
i
\end{array}\right) \frac{(-1)^{\imath}}{a+i+1} \sum_{\jmath=0}^{a+\imath}\left(\begin{array}{c}
a+\imath+1 \\
\jmath
\end{array}\right) B_{\jmath} \bar{d}^{a+b+1-\jmath}(n) .
\end{aligned}
$$

Corollary 6.2.4. Let $a$ and $b$ be any even positıve integers. Then for all $n \in \mathbb{N}$, we have

$$
\begin{aligned}
\sum_{\imath=0}^{a}\left(\begin{array}{l}
a \\
i
\end{array}\right) \frac{(-1)^{\imath}}{b+\imath+1} \sum_{\jmath=0}^{b+\imath}\left(\begin{array}{c}
b+\imath+1 \\
\jmath
\end{array}\right) & B_{\jmath} \bar{d}^{a+b+1-\jmath}(n) \\
& =\sum_{\imath=0}^{b}\left(\begin{array}{l}
b \\
\imath
\end{array}\right) \frac{(-1)^{\imath}}{a+\imath+1} \sum_{\jmath=0}^{a+\imath}\left(\begin{array}{c}
a+\imath+1 \\
\jmath
\end{array}\right) B_{\jmath} \bar{d}^{a+b+1-\jmath}(n) .
\end{aligned}
$$

Theorem 6.2.5. Let $b$ be any odd positive integer. Then for all $n \in \mathbb{N}$, we have

$$
\begin{aligned}
\sigma_{b+1}(n) & +\frac{1}{b+1} \sum_{\imath=0}^{b}\left(\begin{array}{c}
b+1 \\
i
\end{array}\right) B_{\imath} \sigma_{b+2-\imath}(n)+\sum_{\imath=1}^{b}\left(\begin{array}{l}
b \\
\imath
\end{array}\right) \frac{(-1)^{\imath+1}}{\imath+2} \sum_{\jmath=0}^{\imath+1}\left(\begin{array}{c}
\imath+2 \\
\jmath
\end{array}\right) B_{\jmath} \sigma_{b+2-\jmath}(n) \\
& =\frac{b+4}{2 b+4} \sigma_{b+2}(n)+\left(\frac{b+1}{12}\right) \sigma_{b}(n)+\frac{1}{b+2} \sum_{\jmath=2}^{\frac{b+1}{2}}\left(\begin{array}{c}
b+2 \\
\jmath
\end{array}\right) B_{2 \jmath} \sigma_{b+2-2 \jmath}(n) .
\end{aligned}
$$




\subsection{Twisted Convolution Sums}

We introduce the idea of a twrst to our convolution sum $S_{a, b}(n)$. Define the sum $S_{a, b}(n, k)$, for odd positive integers $a$ and $b$ and for any $k \in \mathbb{N}$, to be

$$
S_{a, b}(n, k)=\sum_{m<n / k} \sigma_{a}(n-k m) \sigma_{b}(m)
$$

and we denote $S_{a, b}(n, 1)$ by $S_{a, b}(n)$. Following [23], we say that the convolution sum $S_{a, b}(n, k)$ has level $k$, indicating the level of the twist. The most recent investigations of convolution sums involving divisor functions have been centered around varıous twisted convolution sums. (See [26, pp. 207-228].) Thus, we would like to adopt our Lambert series method to investigate certain twisted convolution sums.

$$
\begin{aligned}
& \text { Consider now the power series } \sum_{n=1}^{\infty} q^{n k} \sigma_{a}(n) \text {. Our claim is that } \\
& \qquad \sum_{n=1}^{\infty} q^{n} \sigma_{a}(n) \sum_{n=1}^{\infty} q^{n k} \sigma_{b}(n)=\sum_{n=1}^{\infty} q^{n} \sum_{m<n / k} \sigma_{a}(n-k m) \sigma_{b}(m) .
\end{aligned}
$$

By expanding the left hand side of (6.3.2), each pair $(n, m)$ will be in 1:1 correspondence with the infinite dimensional matrix $A$ with entries

$$
A[\imath, \jmath]=q^{\imath+\jmath k} \sigma_{a}(\imath) \sigma_{b}(\jmath)
$$

Let $n \in \mathbb{N}$, and let $(\imath, \jmath)$ be any pair in $\mathbb{N} \times \mathbb{N}$ satisfying $\imath+\jmath k=n$. Then we have

$$
A[\imath, j]=q^{n} \sigma_{a}(n-\jmath k) \sigma_{b}(\jmath)
$$

We deduce that the coefficient of $q^{n}$ is the sum over all $\jmath \in \mathbb{N}$ satisfying $\jmath=\frac{n-\imath}{k}$, for any $i \in \mathbb{N}$. As $n$ and $k$ are fixed, we conclude that we must have $\jmath<n / k$, that is, 
the coefficient of $q^{n}$ is

$$
\sum_{m<n / k} \sigma_{a}(n-k m) \sigma_{b}(m)
$$

and we conclude our claim by taking this sum over all $n \in \mathbb{N}$.

Observe that if $k=1$ then $S_{a, b}(n)=S_{b, a}(n)$. If $a \neq b$, then this is not true for any other positive integer $k$, that is, for all $n \in \mathbb{N}$, if $k \geq 2$ then

$$
S_{a, b}(n, k)=S_{b, a}(n, k) \text { if and only if } a=b .
$$

Let $k \geq 2$. If $n=1$, then both $S_{a, b}(n, k)$ and $S_{b, a}(n, k)$ are empty sums, and so equal to 0 . So we take $n$ to be any arbitrary integer satısfying $n \geq 2$. By definitıon,

$$
S_{a, b}(n, k)=S_{b, a}(n, k) \Longleftrightarrow \sum_{m<n / k} \sigma_{a}(n-k m) \sigma_{b}(m)=\sum_{m<n / k} \sigma_{b}(n-k m) \sigma_{a}(m)
$$

We may expand each convolution sum into their product of power series, so that $\sum_{m<n / k} \sigma_{a}(n-k m) \sigma_{b}(m)=\sum_{m<n / k} \sigma_{b}(n-k m) \sigma_{a}(m)$ if and only if, for all $q \in \mathbb{C}$ with $|q|<1$, we have

$$
\sum_{n=1}^{\infty} q^{n} \sigma_{a}(n) \sum_{m=1}^{\infty} q^{m k} \sigma_{b}(m)=\sum_{n=1}^{\infty} q^{n} \sigma_{b}(n) \sum_{m=1}^{\infty} q^{m k} \sigma_{a}(m)
$$

We may write each product of power series as the sum over all entries in an $\mathbb{N} \times \mathbb{N}$ matrix. We let $A[\imath, \jmath]=q^{\imath+\jmath k} \sigma_{a}(\imath) \sigma_{b}(\jmath)$, corresponding to the left hand side of (6.3.5) and $B[i, \jmath]=q^{\imath+\jmath k} \sigma_{b}(\imath) \sigma_{a}(\jmath)$, corresponding to the right hand side of (6.3.5). Then (6.3.5) holds for all $q \in \mathbb{C}$ satisfying $|q|<1$ if and only if $A[\imath, j]=B[\imath, \jmath]$ for all $\imath, j \in \mathbb{N}$, which in turn holds if and only if $q^{\imath+\jmath k} \sigma_{a}(\imath) \sigma_{b}(\jmath)=q^{\imath+\jmath k} \sigma_{b}(\imath) \sigma_{(\jmath)}$ for all 
$\imath, \jmath \in \mathbb{N}$. By taking say, $\jmath=1$, we deduce that $\sigma_{a}(\imath)=\sigma_{b}(\imath)$ for all $\imath \in \mathbb{N}$ if and only if $a=b$.

Our task now is to find a suitable odd function $f$ for use in a Lambert series. This function will also be dependent on its level, $k$. Note also that this function must agree with $f(n)=n^{a}$ when we have the level $k=1$. Thus, we introduce the function

$$
F_{k, a}(n)=\left\{\begin{array}{cc}
\left(\frac{n}{k}\right)^{a}, & \text { if } k \mid n \\
0, & \text { otherwise }
\end{array}\right.
$$

where $a$ is any odd positive integer. Note that $F_{k, a}(n)$ is an odd function. When $k$ and $a$ are understood, we write $F(n)$ for $F_{k, a}(n)$. We examine now $\sum_{n=1}^{\infty} u_{n} F(n)$. We expand the geometric series term to find,

$$
\begin{aligned}
\sum_{n=1}^{\infty} u_{n} F(n)= & \sum_{n=1}^{\infty} F(n)\left(q^{n}+q^{2 n}+q^{3 n}+\cdots\right) \\
= & F(1)\left(q+q^{2}+\cdots\right)+\cdots+F(k)\left(q^{k}+q^{2 k}+\cdots\right)+\cdots \\
& +F(2 k)\left(q^{2 k}+q^{2(2 k)}+\cdots\right)+\cdots
\end{aligned}
$$

From the definition of our function, the term $F(n)$ will be nonzero if and only if $k \mid n$. We can see then that

$$
\sum_{n=1}^{\infty} u_{n} F(n)=\sum_{n=1}^{\infty} q^{n k} \sum_{d \mid n} F(n k)=\sum_{n=1}^{\infty} q^{n k} \sigma_{a}(n)
$$

and by rewriting $n$ as $n / k$, we conclude

$$
\sum_{n=1}^{\infty} u_{n} F(n)=\sum_{n=1}^{\infty} q^{n} \sigma_{a}(n / k)
$$

We now wish to use Theorem 3.1.3 with our function $F(n)$, and rewrite the terms 
of this theorem in terms of power series Unfortunately, we encounter significant difficulties in doing so. However, we are able to determine certain terms

Define

$$
T:=\sum_{n=1}^{\infty} F(n) g(n) u_{n}^{2}
$$

From (2.5.3), we have

$$
u_{n}^{2}=\sum_{t=1}^{\infty}(t-1) q^{n t}
$$

so that

$$
\begin{aligned}
T & =\sum_{n=1}^{\infty} F(n) g(n) \sum_{t=1}^{\infty}(t-1) q^{n t} \\
& =\sum_{n=1}^{\infty} q^{n k} \sum_{d \mid n} F(d k) g(d k)\left(\frac{n}{d}-1\right) \\
& =\sum_{n=1}^{\infty} q^{n k} k^{b}\left(n \sigma_{a+b-1}(n)-\sigma_{a+b}(n)\right)
\end{aligned}
$$

and we simplify to conclude

$$
T=\sum_{n=1}^{\infty} q^{n} k^{b-1}\left(n \sigma_{a+b-1}(n / k)-\sigma_{a+b}(n / k)\right)
$$

The term $T$ will be the same as its Theorem 3.3 .1 counterpart when $k=1$. The remaining terms are of a difficult nature to investigate. It is the author's regret that a general formula for a twisted convolution sum of level $k$ can not yet be determined by this Lambert series method. We believe that this method shows promise, and further research should be directed in this area. 


\subsection{A Comparison of $S_{a, b}(n)$ and $S_{a, b}^{*}(n)$}

Ramanujan's paper on the convolution sum $S_{a, b}^{*}(n)$, for odd positive integers $a$ and $b$, was a seminal work. In fact, both Radoux [21] and Grosjean [13], [14] were able to deduce recurrence relations for $S_{a, b}^{*}(n)$ based on the ideas contained in that paper. Ramanujan's formula (4.2.1) differs from ours given in Theorem 3.3.1, in that his formula is specific to each convolution sum. That is, given any odd $a$ and $b$, we can determine explicitly the most significant term of $S_{a, b}^{*}(n)$. In fact, as we've seen, this will yield the same term used in $S_{a, b}(n)$. The chief disadvantage of Ramanujan's formula is that it is not explicit, and involves a big-Oh error term.

Our formula given in Theorem 3.3.1. differs in that it does give an explicit formula for $S_{a, b}(n)$, but only in terms of all other convolution sums of weight $a+b$. There may be advantages in using this formula to investıgate the associated error term.

There seems to be plenty of further research required in order to fully explore the ramifications of Theorem 3.3.1, and indeed, the Lambert series method by which we reached our conclusions. We hope to use these methods to derive an explicit formula (most likely a recurrence relation) for the twisted convolution sums $S_{a, b}(n, k)$. It would also be interesting to see if we may relate our recurrence formula to those given by Radoux and Grosjean, similar to Theorem 6.1.2. Further, the book [26], recently published, details many identities involving divisor functions, convolution sums and twisted convolution sums. We would like to explore all these identities and revisit them with an eye towards using our Lambert series method. 


\section{Bibliography}

[1] Lars Ahlfors. Complex Analysıs. McGraw-Hill, Inc., New York, 1979.

[2] Ayse Alaca, Saban Alaca, Erin McAfee, and Kenneth S. Williams. Lambert series and Liouville's identities. Dissertationes Mathematıcae, 445, 2007.

[3] Tom M. Apostol. Introduction to Analytıc Number Theory. Springer-Verlag, New York, 1976.

[4] Tom M. Apostol Modular Functions and Dirichlet Series in Number Theory. Springer-Verlag, New York, 1990.

[5] Jordan Bell. A summary of Euler's work on the pentagonal number theorem. Archive for History of Exact Scıences, 3:301-373, 2010.

[6] Bruce B. Berndt and Ae Ja Yee. Congruences for the coefficients of quotients of Eisenstein series Acta Arthmetıca, 104:297-308, 2002.

[7] M. Besge. Extrait d'une lettre de $\mathrm{M}$ Besge à M. Liouville. Journal de Mathématıques Pures et Applıquées, 7:256, 1862.

[8] Nathalie Cheng and Kenneth S. Williams. Evaluation of some convolution sums involving the sum of divisor functions. Yokohama Mathematrcal Journal, 1:39$57,2005$.

[9] H. M. Edwards. Rıemann's Zeta Functıon. Dover Publications, Inc., Mineola, N.Y., 1974.

[10] J. W. L. Glaisher. On the square of the series in which the coefficients are the sums of the divisors of the exponents. The Messenger of Mathematıcs, 14:156163,1884 .

[11] J. W. L. Glaisher. Expressions for the first five powers of the series in which the coefficients are the sums of the divisors of the exponents. The Messenger of Mathematrcs, 15:33-36, 1885.

[12] J. W. L. Glaisher. On certain sums of products of quantities depending upon the divisors of a number. The Messenger of Mathematzcs, 15:1-20, 1885. 
[13] Carl C. Grosjean An infinite set of recurrence formulae for the divisor sums. I. Bulletın de la Société Mathématıque de Belgrque, 29:3-49, 1977.

[14] Carl C. Grosjean. An infinite set of recurrence formulae for the divisor sums. II Bulletın de la Socrété Mathématıque de Belgrque, 29:95-138, 1977.

[15] Heekyoung Hahn. Convolution sums of some functions on divisors. Rocky Mountain Journal of Mathematics, 5:1593-1622, 2007.

[16] G. H. Hardy, P. V. Seshu Aiyar, and B. M. Wilson, editors. Collected Papers of Srınıvasa Ramanujan. Chelsea Publishing Company, 1962

[17] James G. Huard, Zhiming M. Ou, Blair K. Spearman, and Kenneth S. Williams. Elementary evaluation of certain convolution sums involving divisor functions. In M.A. Bennett, B.C. Berndt, N. Boston, H.G. Diamond, A.J. Hildebrand, and W.Philipp, editors, Number Theory for the Millenıum II, pages 229-274, Natick, Massachusetts, 2003. A. K. Peters.

[18] D. B. Lahiri. On Ramanujan's function $\tau(n)$ and the divisor function $\sigma_{k}(n)$. I. Bulletın of the Calcutta Mathematrcal Socrety, 38:33-52, 1946.

[19] D. B. Lahiri. On Ramanujan's function $\tau(n)$ and the divisor function $\sigma_{k}(n)$. II. Bulletin of the Calcutta Mathematrcal Society, 39:193-206, 1947.

[20] Jesper Lützen. Joseph Luouville 1809-1882: Master of Pure and Applied Mathematıcs. Springer-Verlag, Berlin/Heidelberg, 1998.

[21] Christian Radoux. Une nouvelle formule de récurrence pour les sommes de Ra-

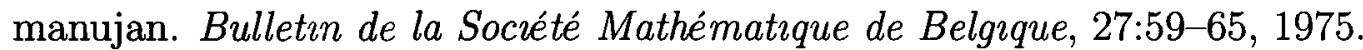

[22] Srinivasa Ramanujan. On certain arithmetical functions. Transactions of the Cambridge Phılosophıcal Socıety, 22:159-184, 1916.

[23] Emmanuel Royer. Evaluating convolution sums of the divisor function by quasimodular forms. International Journal of Number Theory, 2:231-261, 2007.

[24] Walter Rudin. Prıncıples of Mathematıcal Analysıs. McGraw-Hill, Inc., 1976.

[25] Nils-Peter Skoruppa. A quick combinatorial proof of Eisenstem series identities. Journal of Number Theory, 43:68-73, 1993.

[26] Kenneth S. Willams. Number Theory in the Spirit of Liouville. Cambridge University Press, 2011. 